\title{
具有聚集诱导发光特性的含氟有机金属配合物的研究进展
}

\author{
秦成远 ${ }^{a}$ 苗金玲 ${ }^{b}$ 聂 永*,a 刘 威 ${ }^{a}$ \\ 高迎 ${ }^{a}$ 李天瑞 ${ }^{a}$ 蒋绪川*,a \\ ( ${ }^{a}$ 济南大学智能材料与工程研究院 济南 250022) \\ ( ${ }^{b}$ 济南大学化学化工学院 山东省氟化学化工材料重点实验室＼cjkstart济南 250022)
}

\begin{abstract}
摘要 近年来, 具有聚集诱导发光(aggregation-induced emission, AIE)特性的化合物由于在聚集态或固态发光量子效率 较高而受到很多关注. 含氟的功能化合物, 由于氟原子的存在, 往往具有独特的结构和物理、化学及生物学性质. 根据 中心金属和配体的种类, 汇总了具有 AIE 性质的且含有氟代配体的有机金属配合物的研究进展. 这些含氟有机金属配 合物主要是铱、铂、金配合物, 在发光器件、化学传感、细胞成像、数据存储等方面具有潜在应用. 还简要讨论了相 关研究的发展前景.
\end{abstract}

关键词＼cjkstart聚集诱导发光; 氟; 环金属配合物; 有机金属配合物; 磷光

\section{Advances in Fluorinated Organometallic Complexes with Aggregation-Induced Emission Characteristics}

\author{
Qin, Chengyuan $^{a} \quad$ Miao, Jinling ${ }^{b} \quad$ Nie, Yong ${ }^{*, a} \quad$ Liu, Wei $^{a}$ \\ Gao, Ying $^{a} \quad$ Li, Tianrui $^{a} \quad$ Jiang, Xuchuan*,a \\ $\left({ }^{a}\right.$ Institute for Smart Materials \& Engineering, University of Jinan, Jinan 250022) \\ ( ${ }^{b}$ Shandong Provincial Key Laboratory of Fluorine Chemistry and Chemical Materials, School of Chemistry and \\ Chemical Engineering, University of Jinan, Jinan 250022)
}

\begin{abstract}
In recent years, the compounds with aggregation-induced emission (AIE) characteristics have received increasing attention because they exhibit much higher luminescence quantum yields in aggregation or solid states than in solution. Due to the presence of the fluorine atom(s), fluorinated functional compounds generally have unique structures, and physical, chemical and biological properties. Herein, the luminescent organometallic complexes with AIE characteristics having fluorine-containing ligands are summarized, according to the types of central metal atoms and ligands. Such organometallic complexes are mainly those of iridium, platinum and gold, with potential applications in light-emitting devices, chemical sensing, cell imaging and data storage, etc. The prospects of the relevant studies are also discussed.
\end{abstract}

Keywords aggregaion-induced emission; fluorine; cyclometalated complex; organometallic complex; phosphorescence

发光有机金属配合物因其独特、优异的性质被广泛 关注 ${ }^{[1-3]}$, 在光电器件、化学传感及生物成像等领域具有 重要的研究和应用价值 ${ }^{[4-6]}$. 尽管大多数发光有机金属 配合物在分子状态下具有较高的发光效率, 但在固态等 聚集态通常发光较弱, 发生聚集发光猝灭(AggregationCaused Quenching, ACQ)现象(图 1), 因而限制了其实际 应用 ${ }^{[1,3]} .2001$ 年, 唐本忠等提出聚集诱导发光(Aggre-
gation-Induced Emission, AIE)的概念 ${ }^{[7]}$, 解决了传统的 有机发光材料的 ACQ 问题(图 1), 拓宽了发光材料的范 围和应用领域 ${ }^{[8-12]}$. AIE 化合物的发光机制, 主要有分子 内旋转受限 (Restriction of Intramolecular Rotations,

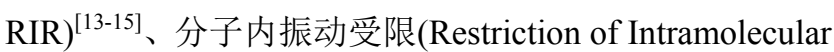
Vibrations, RIV $)^{[15-16]}$ 和分子内运动受限 (Restriction of Intramolecular Motions, RIM)等 ${ }^{[16-18]}$. 典型的 AIE 化合物

\footnotetext{
* Corresponding authors. E-mail: chm_niey@ujn.edu.cn; ism_jiangxc@ujn.edu.cn Received July 5, 2020; revised August 18, 2020; published online September 15, 2020.

Project supported by the Natural Science Foundation of Shandong Province (Nos. ZR2017LB008, ZR2020MB001), the Science and Technology Program of University of Jinan (No. XKY1906) and Shandong Shenna Smart Advanced Materials Co., Ltd.

山东省自然科学基金(Nos. ZR2017LB008, ZR2020MB001)、济南大学科技计划(No. XKY1906)和山东莘纳智能新材料有限公司资助项目.
} 
包括多芳基取代杂环化合物、多芳基乙烯、分子内电荷 转移化合物、聚合物、(有机) 金属配合物以及含氢键体 系等 ${ }^{[13-19]}$. AIE 化合物大多为纯有机物, 具有 AIE 特性 的有机金属配合物也有一些报道 ${ }^{[20-23]}$. AIE 有机金属配 合物的发光机理, 主要是 RIR 或 RIM, 有些体系中金 属一金属相互作用也有贡献. 由于含有重金属原子, 发 光有机金属配合物可能同时利用单线态和三线态激子, 发射荧光或磷光, 或同时发射荧光和磷光. 过渡金属配 合物的发光常包含金属到配体的电荷转移跃迁 (metal-to-ligand charge-transfer transition, MLCT), 由于 重金属原子促进的自旋一轨道耦合作用, 该类的型跃迁 能促进磷光发射. 2008 年, 李富友、刘志攀、黄春辉等 ${ }^{[24]}$ 发现部分铱配合物在固态下由于分子堆积形成分子间 $\pi-\pi$ 相互作用, 导致激发态由溶液中的弱发光 ${ }^{3} \mathrm{LC}$ (ligand centered, 配体中心)态转变为聚集态时的 ${ }^{3}$ MLLCT (metal-to-ligand-ligand charge transfer, 金属到配体-配体 的电荷转移)跃迁态, 具有较强的磷光发射, 称之为聚 集诱导磷光发射 (aggregation-induced phosphorescence emission, AIPE). 研究表明, 部分 AIE 有机金属化合物 的发光属于 AIPE 范围. 相对于数量庞大、性能突出的 AIE 荧光有机物, AIE 磷光有机金属配合物在材料类型、 发光机制和应用研究等方面都需要进一步加强 ${ }^{[25]}$.

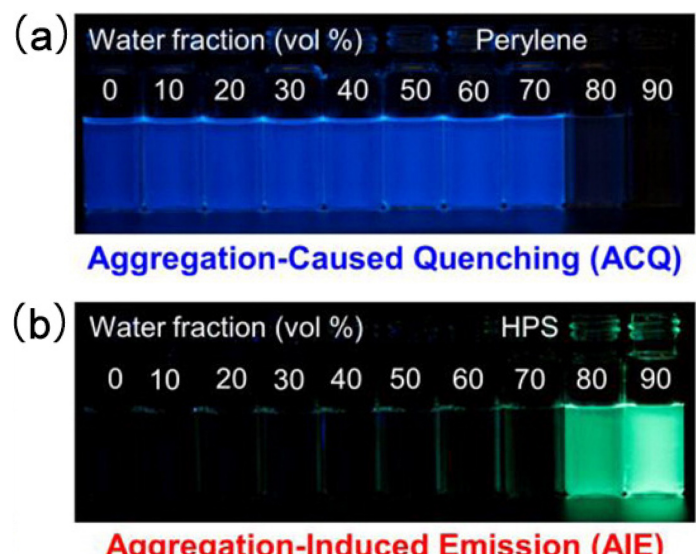

图 1 (a)在四氢呋喃/水中随含水量增加的菲 $(20 \mu \mathrm{mol} / \mathrm{L})$ 的 $\mathrm{ACQ}$ 效应和荧光照片, 以及(b)六苯基噻咯 $(20 \mu \mathrm{mol} / \mathrm{L})$ 的 $\mathrm{AIE}$ 效应 ${ }^{[14]}$

Figure 1 ACQ effect and fluorescence photographs of perylene $(20 \mu \mathrm{mol} / \mathrm{L})(\mathrm{a})$, and AIE effect of hexaphenylsilole $(20 \mu \mathrm{mol} / \mathrm{L})$ (b) in THF/water mixtures with increasing water fraction

另一方面, 氟原子具有独特的性质(较小的原子半 径与大的电负性), 氟代是一种有效的化学修饰手 段 ${ }^{[26-27]}$. 将氟原子引入分子中, 往往会赋予分子特殊结 构和物理、化学及生物学性质, 故氟代在材料与化学领 域被广泛研究. 在发光材料领域, 引入氟原子一般使材 料稳定性提高, 发光性能更理想 ${ }^{[28-29]}$. 将含氟配体引入
到发光金属配合物中，可以增强配合物分子间的相互作 用, 使固态发光寿命及发光量子效率更优良 ${ }^{[28-29]}$, 可能 赋予化合物诸如力致发光变色(mechanochromic luminescence, MCL)及热致发光变色等刺激响应性 ${ }^{[30]}$, 使发 光有机金属配合物具有独特的光物理特性和更多的潜 在应用 ${ }^{[31]}$.

含氟的 AIE 有机金属配合物也有一些报道, 但其数 量还相对较少. 汇总和分析已知的含氟 AIE 有机金属配 合物的结构特点、发光性能和潜在应用, 将有助于 AIE 有机金属配合物和含氟光功能化合物的研究. 关于 AIE 金属配合物，已有一些相关的综述 ${ }^{[20-23]}$, 但目前还没有 综述专门讨论氟原子的引入对 AIE 有机金属配合物的 影响. 最近, 我们汇总了具有 AIE 性质的有机氟化合 物 ${ }^{[32]}$. 在此基础上, 根据中心金属和配体的种类，综述 了具有 AIE 特性的、含有氟代配体的有机金属配合物的 研究进展, 并简要讨论相关研究的发展前景. 为了方便, 本文不讨论配体不含氟而含氟部分仅为三氟醋酸根、三 氟甲磺酸根、 $\mathrm{BF}_{4}^{-}$以及 $\mathrm{PF}_{6}^{-}$等阴离子的 $\mathrm{AIE}$ 有机金属配 合物暂不予讨论.

\section{1 含氟铱配合物}

铱配合物具有发光效率高、发光颜色可调、热稳定 性好、发光寿命长和斯托克斯位移大等优点 ${ }^{[38-40]}$. 发光 铱配合物通常是三价铱离子与三个双齿配体组成的具 有八面体结构的配合物, 一般是均一配合物 $\left[\operatorname{Ir}\left(\mathrm{C}^{\wedge} \mathrm{N}\right)_{3}\right]$ 或混配配合物 $\left[\operatorname{Ir}\left(\mathrm{C}^{\wedge} \mathrm{N}\right)_{2}\left(\mathrm{~L}^{\wedge} \mathrm{X}\right)\right]$ (其中, $\mathrm{C}^{\wedge} \mathrm{N}$ 称为主配体, $\mathrm{L}^{\wedge} \mathrm{X}$ 称为辅助配体), 也称为环金属配合物 (cyclometalated complex), 其光物理性质主要取决于其主配体 的性质和共轭程度 ${ }^{[38-41]}$. 铱配合物是目前报道的 AIE 金 属配合物中数量最多的. 在含氟配体的 AIE 铱配合物 中, 主配体主要有氟代苯基吡啶、氟代苯基吡唑和氟代 苯基咪唑三种. 表 1 汇总了含氟 AIE 有机铱配合物的光 物理性质和潜在应用.

\section{1 含氟代苯基吡啶配体的铱配合物}

含氟代苯基吡啶配体的铱配合物, 既有中性的, 也 有阳离子型的; 从核数来看, 既有单核的, 也有双核的; 既有同双核的，也有异双核的. 2008 年，Park 等 ${ }^{[42]}$ 合成 了含(2,4-二氟苯基)吡啶配体的铱配合物 1 和 2.1 和 2 具有聚集诱导磷光特性, 在固态下表现出强的磷光发 射. 通过实验及理论计算等多种手段研究, 认为其磷光 是由其主配体及辅助配体的芳环在固态下的 RIR 机制 导致, 这是最早报道的具有 AIPE 特性的含氟铱配合物. 相对于含有苯基吡啶配体的类似物, 1 和 $\mathbf{2}$ 的发射光谱 发生明显蓝移, 发光量子效率也更高. 2010 年, Do 等 ${ }^{[43]}$ 
表 1 含氟铱配合物的光物理性质及潜在应用

Table 1 Photophysical properties and potential applications of fluorinated iridium complexes

\begin{tabular}{|c|c|c|c|c|c|c|}
\hline 化合物 & 吸收峰 ${ }^{a} / \mathrm{nm}$ & 发射峰 ${ }^{b} / \mathrm{nm}$ & 发光量子效率 ${ }^{b} / \%$ & 发光寿命 & 潜在应用 & 文献 \\
\hline 1 & - & 563 & 53 & - & - & {$[42]$} \\
\hline 2 & - & 596 & 24 & - & - & {$[42]$} \\
\hline 3 & $254,290,330,388,437,465$ & 605 & - & $23 \mathrm{~ns}^{a}$ & OLED, 传感器 & {$[43]$} \\
\hline 4 & $253,290,328,388,435,465$ & 597 & - & $22 \mathrm{~ns}^{a}, 67 \mathrm{~ns}^{b}$ & OLED, 传感器 & {$[43]$} \\
\hline 6 & $\begin{array}{l}259,281,327,369,388,435 \\
465\end{array}$ & 597 & - & $22 \mathrm{~ns}^{a}, 56 \mathrm{~ns}^{b}$ & OLED, 传感器 & [43] \\
\hline 7 & $268,350,430$ & $441,472,506$ & 5.41 & - & - & {$[44]$} \\
\hline 8 & $255,338,369,430$ & $448,476,507$ & 41.43 & - & - & [44] \\
\hline 9 & $255,339,367,419$ & $443,478,507$ & 10.65 & - & - & {$[44]$} \\
\hline 10 & $262,337,369,419$ & 481 & 10.95 & - & - & {$[44]$} \\
\hline 11 & $275,371,430$ & $460,493,529$ & 5.77 & - & - & [44] \\
\hline 12 & $254,307,370,469$ & 461,488 & 7.25 & $2.00 \mathrm{~ns}^{b}$ & $\mathrm{Hg}(\mathrm{II})$ 传感器 & {$[45]$} \\
\hline 13 & $253,302,371,450$ & 461,488 & 6.62 & $3.38 \mathrm{~ns}^{b}$ & Hg(II)传感器 & {$[45]$} \\
\hline 14 & $253,302,271,450$ & 461,488 & - & - & - & [45] \\
\hline 15 & $264,297,337,406$ & 505 & 11 & $9.4 \mu \mathrm{s}^{b}$ & $\begin{array}{l}\text { 探针、发光材料和数 } \\
\text { 据保护 }\end{array}$ & [46] \\
\hline 16 & $268,298,337,458$ & 569 & 12 & $9.7 \mu \mathrm{s}^{b}$ & $\begin{array}{l}\text { 探针、发光材料和数 } \\
\text { 据保护 }\end{array}$ & {$[46]$} \\
\hline 17 & $265,298,350,406$ & 511 & 28 & - & 潜在指纹检测 & {$[47]$} \\
\hline 18 & $264,297,335,487$ & 660 & 20 & $7.07 \mathrm{~ms}^{b}$ & 线粒体靶向探针 & {$[48]$} \\
\hline 19 & $263,296,331,347$ & 593 & 12 & $5.72 \mathrm{~ms}^{b}$ & 线粒体靶向探针 & [48] \\
\hline 20 & 245,351 & 550 & 7.4 & - & TNT-磷光探针 & [49] \\
\hline 21 & 266,359 & 584 & 4.4 & - & 光动力疗法 & {$[50]$} \\
\hline 22 & 374 & 544 & 6.8 & $4.24 \mu \mathrm{s}^{b}$ & 线粒体靶向探针 & {$[51]$} \\
\hline 23 & - & 452,486 & 52 & $1.4 \mu \mathrm{s}^{b}(486 \mathrm{~nm})$ & - & {$[52]$} \\
\hline 24 & $242,309,360$ & 625 & 6 & $27.95 \mu \mathrm{s}^{b}$ & 线粒体靶向探针 & {$[53]$} \\
\hline 25 & - & 537 & - & - & 光学记录, 压力感应 & {$[54]$} \\
\hline 26 & - & 461 & - & $1.20 \mu \mathrm{s}^{b}$ & - & {$[55]$} \\
\hline 27 & - & 467 & - & $0.29 \mu \mathrm{s}^{b}$ & 化学传感 & {$[55]$} \\
\hline 28 & - & 512 & - & $1.38 \mu \mathrm{s}^{b}$ & - & {$[55]$} \\
\hline 29 & - & - & 23.6 & - & - & {$[56]$} \\
\hline 30 & $249,319,357$ & 612 & 31 & $0.18 \mu \mathrm{s}^{a}$ & 光学记录 & [57] \\
\hline 31 & $253,322,366$ & 627 & 24 & $0.17 \mu \mathrm{s}^{a}$ & 光学记录 & {$[57]$} \\
\hline $32 \mathrm{a}$ & - & 479 & 33.3 & $0.023 \mu \mathrm{s}^{a}, 1.16 \mu \mathrm{s}^{b}$ & 信息存储，数据保护 & {$[58]$} \\
\hline 32b & - & 498 & 5.9 & $0.028 \mu \mathrm{s}^{a}, 1.20 \mu \mathrm{s}^{b}$ & 信息存储, 数据保护 & [58] \\
\hline $32 \mathrm{c}$ & - & 516 & 8.4 & $0.038 \mu \mathrm{s}^{a}, 0.28 \mu \mathrm{s}^{b}$ & 信息存储，数据保护 & {$[58]$} \\
\hline 33 & 607 & 529 & 75 & $0.67 \mu \mathrm{s}^{b}$ & 传感, 数据记录 & [59] \\
\hline 34 & - & $\approx 500$ & - & - & TNP 检测 & {$[60]$} \\
\hline 35 & $372,402,457$ & 469,500 & 11 & $1.82 \mu \mathrm{s}^{b}(500 \mathrm{~nm})$ & 防伪 & [61] \\
\hline 36 & $355,378,459$ & 470,501 & 55 & $2.80 \mu \mathrm{s}^{b}(501 \mathrm{~nm})$ & 防伪 & [61] \\
\hline 37 & $365,384,442$ & 456,486 & 57 & $4.15 \mu \mathrm{s}^{b}(486 \mathrm{~nm})$ & 防伪 & [61] \\
\hline 38 & $360,379,443$ & 452,480 & 52 & $4.55 \mu \mathrm{s}^{b}(480 \mathrm{~nm})$ & 防伪 & [61] \\
\hline 39 & - & - & 10.6 & - & 指纹检测 & {$[62]$} \\
\hline 40 & 275,330 & 716 & 12 & $1.93 \mu \mathrm{s}^{b}$ & OLED & [63] \\
\hline
\end{tabular}

${ }^{a}$ Solution; ${ }^{b}$ Solid; - : Unreported.

合成了铱配合物 $3 \sim 6$. 这几个配合物均具有优异的发 光性能, 在有机发光二极管(Organic Light-Emitting Diode, OLED)及传感等领域具有应用潜力. 其中, 4 为双核 铱配合物, 6 为双核铱铂配合物, 它们有相似的发光行 为. 6 的铂核部分在固态下发光猝灭, 所以 4 和 $\mathbf{6}$ 的固态 发光行为主要由金属铱与苯基吡啶配体的 MLLCT 机理
导致. 2014 年, Laskar 等 ${ }^{[44]}$ 合成了一系列铱配合物 7 11，这些配合物具有 AIE 特性及不同的固态发光波长. 7 10 的固态发光波长相对于其苯基吡啶配体类似物发 生明显的蓝移，其中具有三(4-氟苯基)膦配体的 7 的发 射光谱, 相对于膦配体不含氟的 $\mathbf{8} \sim \mathbf{1 0}$, 固态发光波长 也发生一定程度的蓝移(表 1). 铱配合物 11 为配合物 


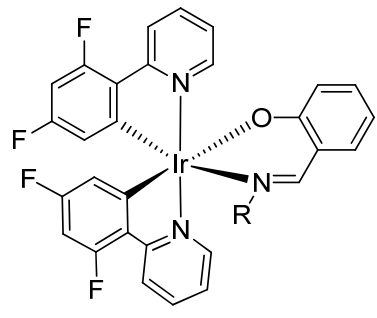<smiles></smiles>

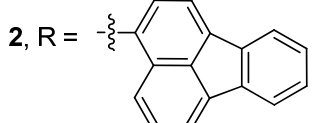

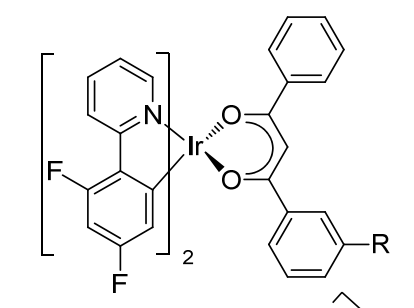

3, $\mathrm{R}=\mathrm{H}$

$4, R=$<smiles>CC(C)(C)C(=O)/C=C(\O)c1ccccc1</smiles>

$6, R=$<smiles>CN1Cc2ccccc2P12(OC(C)(C)C)OCC(CC(C)(C)C)(c1ccccc1)O2</smiles>

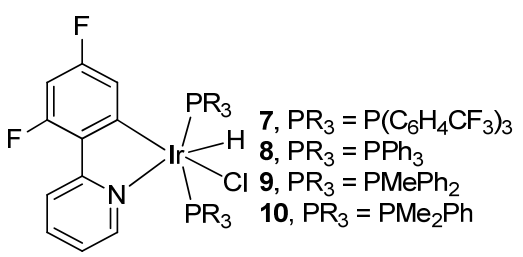

7 在苯基吡啶配体上不含氟的类似物，二者具有相同的 三(4-三氟甲基苯基)膦配体，其固态发光波长比 $8 \sim \mathbf{1 0}$ 的含苯基吡啶配体类似物发生 10 40 nm 的蓝移. 这说 明, 在铱配合物的主配体及辅助配体中引入氟原子或含

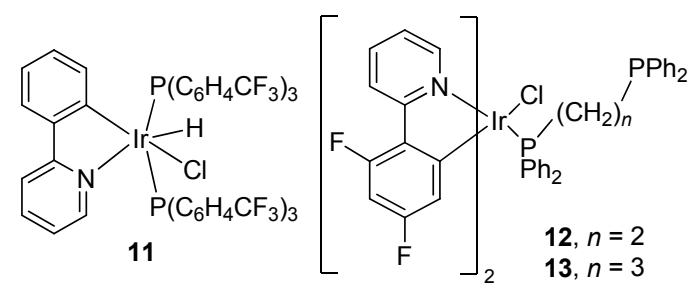

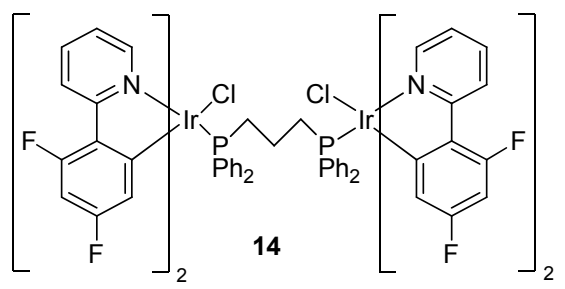

氟取代基，都可能对其固态发光波长产生影响. 同年, Laskar 等 ${ }^{[45]}$ 还报道了铱配合物 12 14. 其中, 12 和 13 配体上的非配位磷原子可以选择性地与 $\mathrm{Hg}^{2+}$ 离子结合, 导致其发光猝灭，从而有效地检测 $\mathrm{Hg}^{2+}$ 离子.

朱红军和刘睿等 ${ }^{[46]}$ 报道的铱配合物 15 具有 AIPE 特性, 同时显示可逆的 MCL 与溶剂响应特性. 基于此, 他们设计了一种用于信息加密和解密的技术, 如图 2 所 示. 将滤纸在染料 $(E)-4$-磺酸-4'-二甲氨基氮二苯乙烯 $(\mathrm{SDMAB})$ 的二氯甲烷溶液中浸泡，得到附有该染料的 黄色滤纸, 在 $365 \mathrm{~nm}$ 处用作背景. 在加密阶段, 将浸在 专门制作的 “墨水” (15 的乙醇溶液)中的 “印章” (刻有 字母“NJ TECH”)印在黄色滤纸上. 由于颜色相似, 干燥 后肉眼无法观察到字母, 即记录的数据被加密. 在解密 阶段, 在 $365 \mathrm{~nm}$ 紫外灯照射下, 将滤纸暴露于二氯甲烷 气氛 $10 \mathrm{~min}$, 可以清楚地呈现字母“NJ TECH”的绿色荧 光. 含氟铱配合物 $16^{[46]}$ 为 15 的类似物, 其主配体为 2-(3,5-二氟苯基)喹啉. 15 和 16 具有相似的磷光寿命及 发光量子效率, 其固态发光波长相对于其含苯基吡啶配 体的类似物有明显的蓝移. 2018 年, 朱红军和刘睿 等 [47]合成了铱配合物 17. 基于其 AIPE 特性及亲脂性, 17 可以用于指纹的显现, 在 $5 \mathrm{~min}$ 内提供准确的指纹定量 识别数据, 在刑侦等领域具有应用前景. 最近, 朱红军 和刘睿等 ${ }^{[48]}$ 报道了具有 AIPE 性质的铱配合物 18 和 19 , 发现这两个配合物能在线粒体中选择性积累, 可应用于 活细胞的线粒体成像. 配合物 15、17 19 具有较相似的 结构, 其中 17 比 15 的主配体具有更多的氟原子取代, 其固态发光量子效率相比 15 更高, 发射光谱也发生蓝 移(表 1). 18 的主配体比 15 多了酯基取代基，其固态发 射峰(660 nm)比 15 (505 nm)发生红移. 通过对比 18 和 19 的光物理性质(表 1)可以发现, 将氟原子以不同的形 式引入到铱配合物的配体中，能导致不同的发光性能.

2014 年, Reddy 等 ${ }^{[49]}$ 合成了铱配合物 20. 20 具有 AIE 性质，可以用于三硝基甲苯(TNT)的检测，其发光 随着 TNT 的含量增加而猝灭. 2017 年，巢辉等 ${ }^{[50]}$ 合成了 铱配合物 21. 21 具有显著的 AIE 特性及线粒体靶向性, 可选择性地积聚在线粒体中. 21 还具有光动力活性, 可 作为光敏剂应用于双光子吸收-光动力疗法(Two-photon Absorbing-Photodynamic Therapy, TPA-PDT; 图 3). 同 年, 巢辉等 ${ }^{[51]}$ 合成了具有 AIPE 特性的铱配合物 22, 相 对于含苯基吡啶配体的类似物, 22 的发射光谱发生明显 的蓝移，也具有更长的磷光寿命. 同时, 22 具有聚集诱 导的双光子发光(AITPE, aggregation-induced two-photon emission)特性，在聚集态下具有优异的双光子发射性质 及亲脂性，在水中聚集并靶向线粒体，可用于单层细胞 和多细胞球体(multicellular spheroids)中线粒体靶向的 

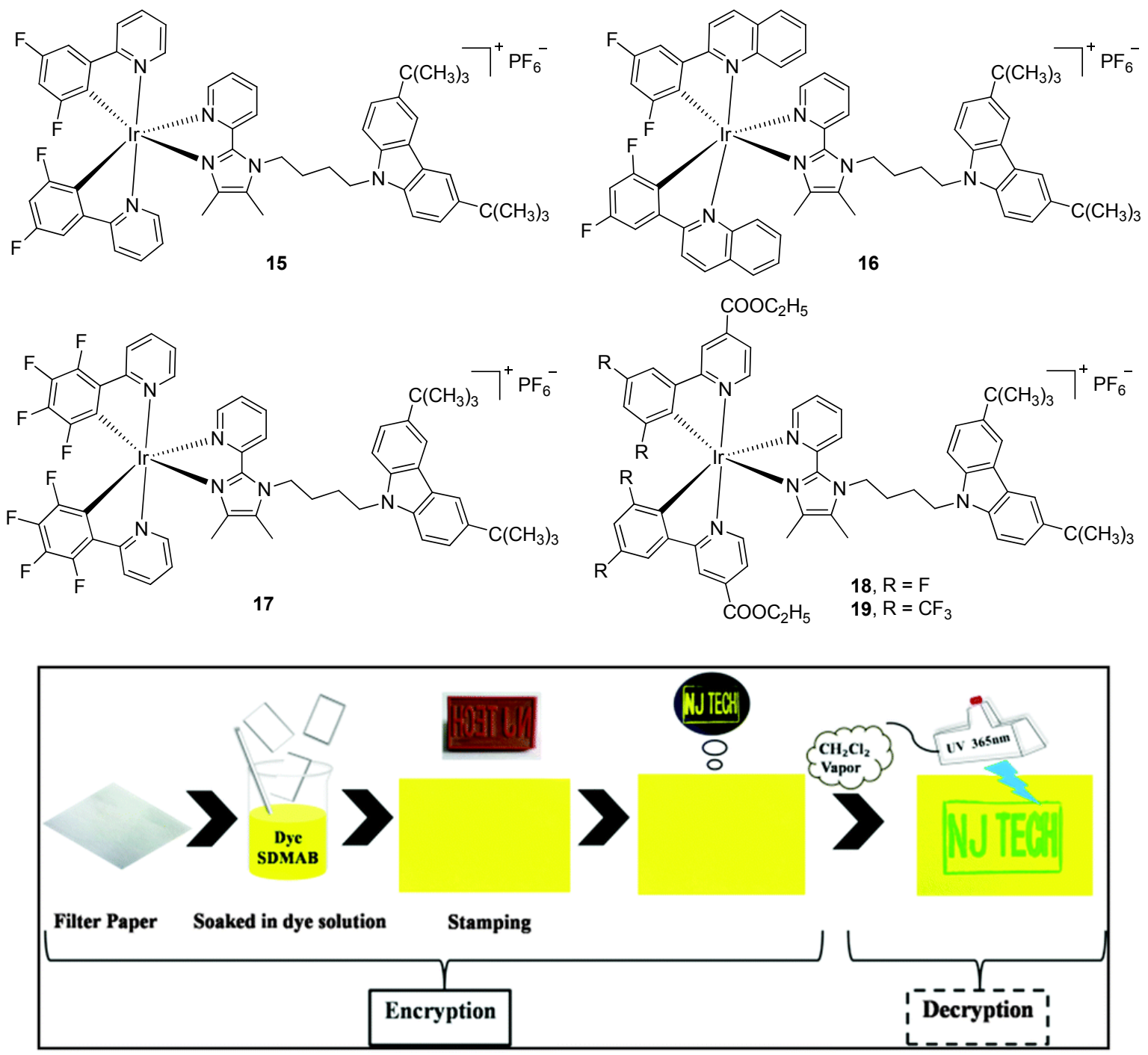

图 2 配合物 15 用于数据加密和解密示意图 ${ }^{[46]}$

Figure 2 Procedures and photographs of data encryption and decryption of complex $\mathbf{1 5}^{[46]}$<smiles></smiles>

20

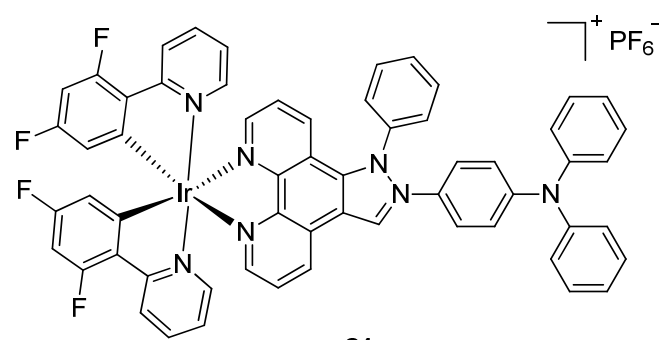

21

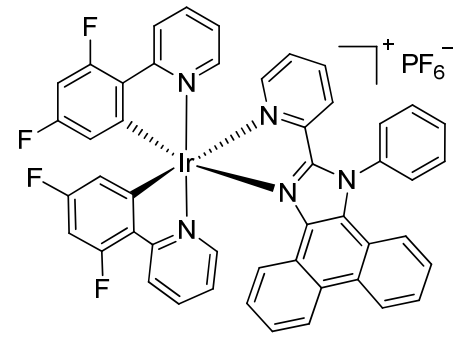

22
双光子 PDT 及细胞成像. 2019年, De Cola 等 ${ }^{[52]}$ 合成了具 有 AIE 发蓝光特性的铱配合物 23. 由于结构中烷基链末 端 $\mathrm{OSO}_{3}^{-}$基团的负电荷增强了晶态下烷基链的刚性, 23 具有较强的固态发光, 而与 23 具有相似结构的、末端无 $\mathrm{OSO}_{3}^{-}$基团的类似物, 在固态及晶态下发光猝灭. 最近, 朱东霞等 ${ }^{[53}$ 报道了具有聚集发光增强 [aggregationinduced enhanced emission (AIEE), 也称为 aggregationenhanced emission (AEE)] 特性的双核铱配合物 24. 24 的
固体发射峰 $(625 \mathrm{~nm})$ 比其不含氟的类似物的发射峰发生 蓝移，也具有更长的发光寿命. 同时, 24 还具有 MCL 特 性.

上述铱配合物，其稀溶液发光都很弱，而固态发光 显著增强，这与配合物的配体结构及聚集状态有关. 在 溶液中，配体上的杂环及苯环发生旋转，消耗激发态能 量, 减弱配合物的发光强度. 在固态时, 分子间距减小, 增大了空间位阻，阻碍了分子内运动，使发光增强. 配 


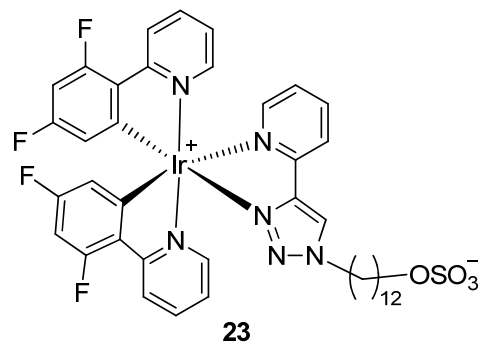

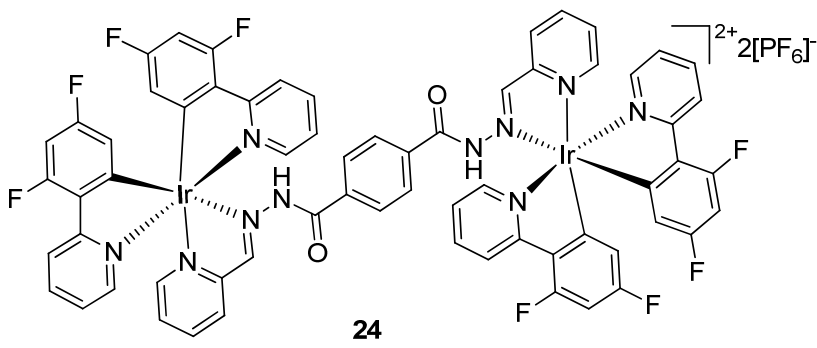

24

Two-photon Laser

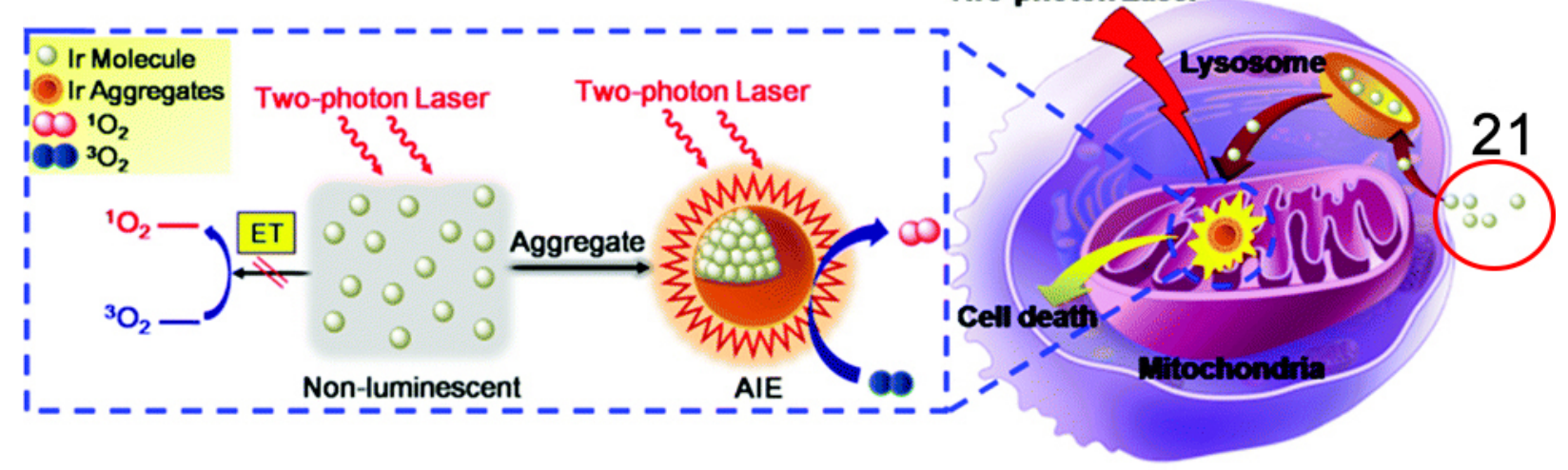

图 3 配合物 21 在线粒体中的 TPA-PDT 示意图 ${ }^{[50]}$

Figure 3 Schematic illustration of lit up TPA-PDT in mitochondria by complex $\mathbf{2 1}^{[50]}$

合物的发射波长等性质, 可以通过改变配体进行调控.

\section{2 含氟代苯基吡唑配体的铱配合物}

含氟代苯基吡唑配体的铱配合物, 主要是阳离子型 的, 中性的较少. 2012 年, 苏忠民等 ${ }^{[54]}$ 合成了含 1-(2,4二氟苯基)吡唑配体的铱配合物 25.25 具有 AIE 及可逆 的 MCL 特性, 其粉末研磨后发光波长从 $537 \mathrm{~nm}$ 红移至 $564 \mathrm{~nm}$ (图 4), 通过加热或再结晶能恢复到原来的发光 波长. 分析发现, 25 的 MCL 特性主要是由于晶态到非晶 态的相变, 这在光学记录及压力传感等领域有潜在应 用.

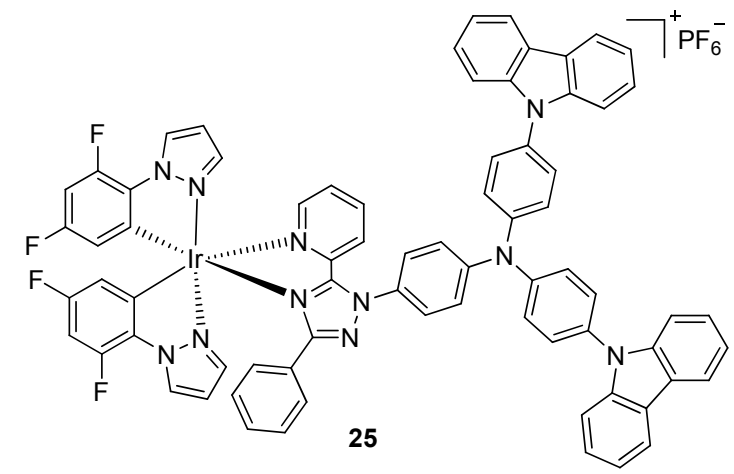

2013 年, 苏忠民等 ${ }^{[55]}$ 合成了含(2,4-二氟苯基)吡唑 配体的铱配合物 $26 \sim 28$, 其中 26 仅表现出 AIE特性, 而 27 和 28 则同时具有 AIE 及可逆的 MCL 特性(图 5). 而 且, 由于其纳米聚集体的发光可以被 TNT 猝灭, 配合物
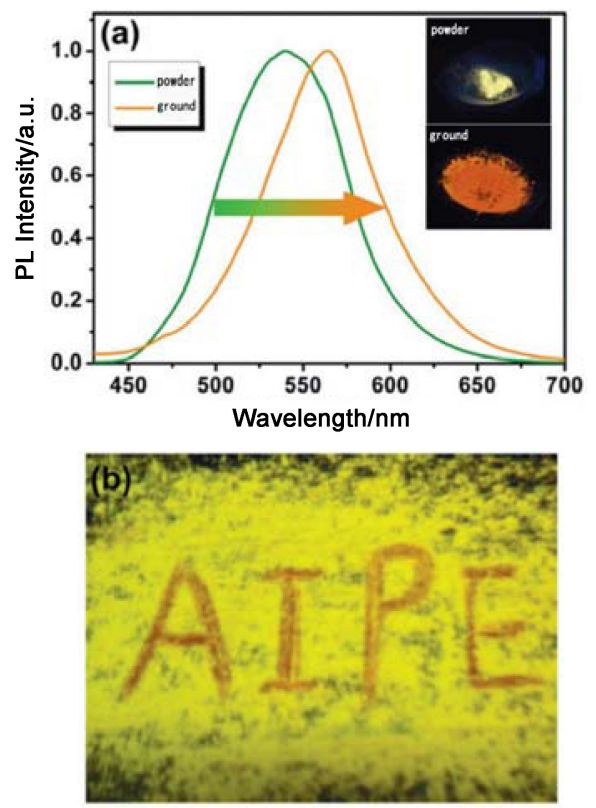

图 4 (a)配合物 $\mathbf{2 5}$ 粉末与研磨后的发射光谱和(b)将配合物 $\mathbf{2 5}$ 的粉末浇铸在滤纸上, 紫外光下用刮刀在室温书写字母 "AIPE",[54]

Figure 4 (a) Emission spectra of complex 25: powder and after being ground, and (b) the powder $\mathbf{2 5}$ was cast on the filter paper and the letters "AIPE" were written with a spatula under UV light at room temperature ${ }^{[54]}$

27 在 TNT 检测等领域具有应用潜力. 2014 年，单国刚 等 ${ }^{[56]}$ 报道了含 $(2,4$-二氟苯基)吡唑配体的铱配合物 29 , 该配合物表现出 AIE 特性, 固态下发蓝色苂光. 


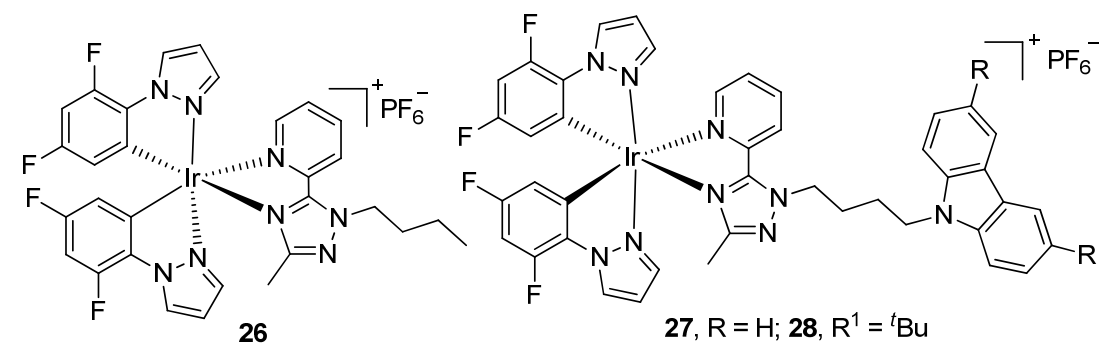

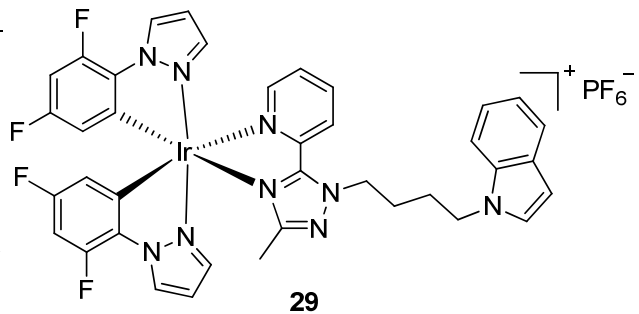
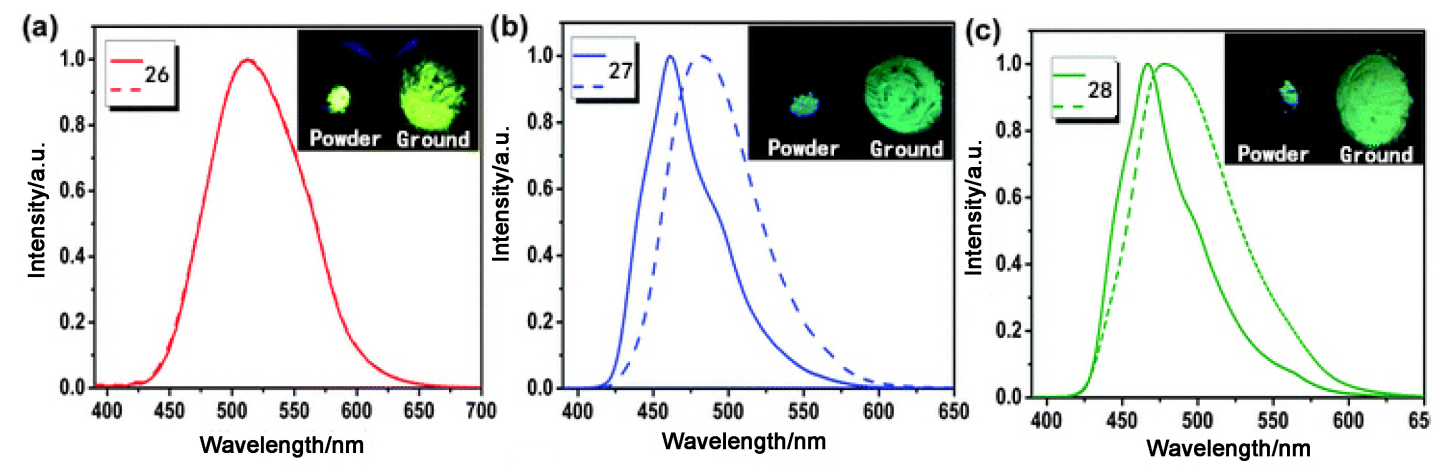

图 5 配合物 26 28 室温研磨前(实线)后(虚线)的发射光谱 ${ }^{[55]}$

Figure 5 Emission spectra of complexes $\mathbf{2 6} \sim \mathbf{2 8}$ in solid state at room temperature before (solid line) and after (dotted line) grinding ${ }^{[55]}$

2015 年, 朱东霞等 ${ }^{[57]}$ 合成了含(2,4-二氟苯基)吡唑 配体的双核铱配合物 30 和 31, 二者均具有可逆的力致 磷光变色行为, 可用于构建可擦写磷光数据记录器件. 晶体结构分析和含时密度泛函(TD-DFT)计算表明, 固 态时分子内运动受限是二者具有 AIE 性质的主要原因. 2018 年, 苏忠民等 ${ }^{[58]}$ 报道了含(2,4-二氟苯基)吡唑配体 的铱配合物 32, 含氟配体的引入使其相对于其含苯基 吡唑配体的类似物, 具有更高的发光量子效率和更明显
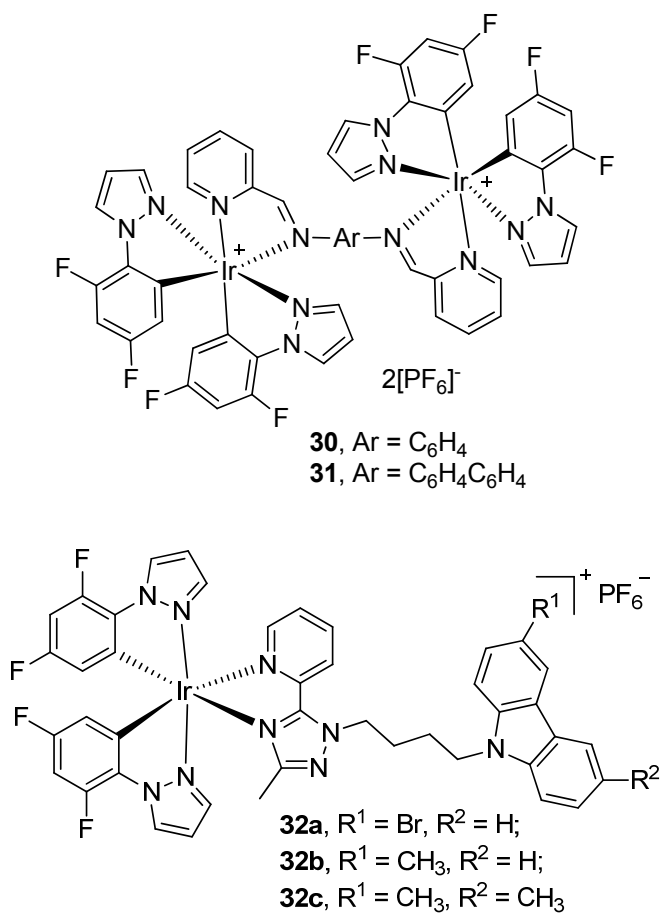

的 AIE 特性，可以用于信息存储和数据保护等领域. 苏 忠民等 ${ }^{[59]}$ 还报道了含有 $(2,4$-二氟苯基)吡唑配体的铱配 合物 33, 与含 1-苯基吡唑配体的类似物相比, 其发射光 谱发生明显的蓝移，分子的能隙也明显增加. 2018 年, 朱东霞等 ${ }^{[60}$ 报道了一系列含 $(2,4-$ 二氟苯基)吡唑配体的 铱配合物 34, 这些配合物具有 AIEE 特性且可用于 2,4,6-三硝基苯酚(TNP)的检测，其发光随 TNP含量的增 加而猝灭.

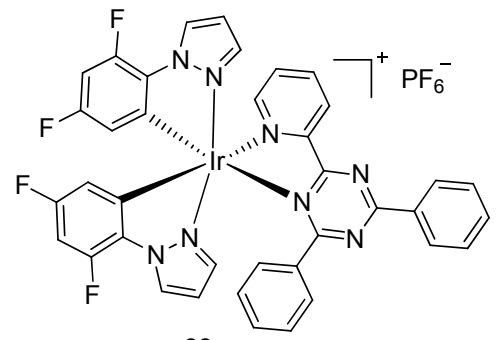

33

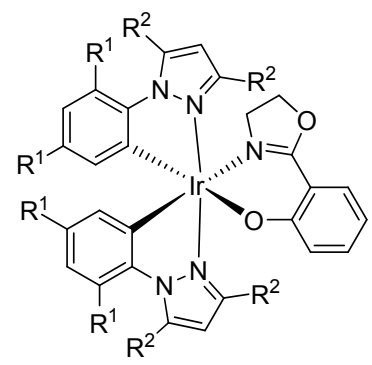

34a, $\mathrm{R}^{1}=\mathrm{F}, \mathrm{R}^{2}=\mathrm{H}$;

34b, $\mathrm{R}^{1}=\mathrm{CF}_{3}, \mathrm{R}^{2}=\mathrm{H}$;

34c, $\mathrm{R}^{1}=\mathrm{CF}_{3}, \mathrm{R}^{2}=\mathrm{CH}_{3}$

上述含氟代苯基吡唑配体铱配合物的 AIE 机理也 
属于其主配体及辅助配体中旋转单元的 RIR 效应. 而 且，单核阳离子型铱配合物 $25 \sim 29$ 以及 32 和 33 的辅助 配体不同, 具有不同的光物理性质, 说明可以通过对辅 助配体进行合理搭配来调控配合物的发光性能. 例如 32a 32c, 仅通过改变辅助配体上的取代基, 就能改变 整个配合物分子的偶极矩, 从而调节配合物的发射波长 和发光量子效率等性质.

\section{3 含氟代苯基咪唑(噻唑)及其他配体的铱配合物}

含氟代苯基咪唑或其他一些含氟配体的铱配合物 也有报道, 这些铱配合物也因其主配体及辅助配体中的 旋转单元在聚集态的 RIR 机理而表现 AIE 特性. 2018 年, Bryce 等 ${ }^{[61]}$ 报道了具有 AIPE 性质的双核 $\operatorname{Ir}(\mathrm{III})$ 配合物 35 38. 35 38 具有较高的聚集态发光效率, 在防伪等 方面有应用潜力. 35 和 36 具有相似的结构, 仅桥联配体 的含氟基团不同, 含有五氟苯基取代基的 36 的固态发 光效率远高于 $35 ; 37$ 和 38 仅主配体苯环上的氟原子数 目不同，具有较多氟取代的 38 具有比 37 更长的磷光寿 命，这是由于更多氟取代基的引入使配合物的分子间作 用力得到增强. 2018 年, 朱红军和刘睿等 ${ }^{[62]}$ 报道了含有 氟代苯基噻唑配体的铱配合物 39. 39 具有 AIPE 特性及 亲脂性, 可以用于指纹显现, 能提供准确的指纹定量识 别数据且显现时间只需 $1 \mathrm{~min}$, 在刑侦等领域具有应用 前景. Bejoymohandas 等 ${ }^{[63]}$ 报道了具有 AIPE 性质的配合 物 40. 在 40 的晶体结构中, 在苯并噻吩和三氟甲基之 间存在 $\mathrm{C}-\mathrm{F} \cdots \pi$ 相互作用, 且三氟甲基的存在也减少了 分子的扭曲运动, 分子内旋转受限导致了 AIPE 现象. 由于三氟甲基(吸电子基)的引入, 40 的发射峰(716 nm)
比其相同位置为苯基 $(660 \mathrm{~nm})$ 和氢 $(666 \mathrm{~nm})$ 的类似物都 发生红移. 他们还制备了基于 40 的磷光有机发光二极 管(phosphorescent organic light emitting diodes, PhOLEDs), 其外量子效率(External Quantum Efficiency, EQE) 达 $7.29 \%$.

\section{2 含氟铂配合物}

发光铂配合物具有发光寿命长、斯托克斯位移大、 发射波长可调以及光稳定性好等优点 ${ }^{[64]}$, 且铂(II)配合 物的平面构型也有利于分子间聚集. 在铂配合物中引入 含氟配体，可能具有更稳定的分子间堆积结构和可控的 发射波长等特性. 已报道的含氟 AIE 铂配合物中, 铂以 二价为主，一般含有两个双齿或一个三齿氟代螯合配 体, 其类型为 $\mathrm{C}^{\wedge} \mathrm{N}, \mathrm{N}^{\wedge} \mathrm{N}, \mathrm{N}^{\wedge} \mathrm{C}^{\wedge} \mathrm{N}, \mathrm{C}^{\wedge} \mathrm{N}^{\wedge} \mathrm{N}$ 等. 表 2 汇总 了含氟 AIE 铂配合物的光物理性质和潜在应用.

2012 年, 赵强等 ${ }^{[65]}$ 报道了含氟代苯基吡啶配体及 不同 $\mathrm{N}^{\wedge} \mathrm{O}$ 配体的铂(II)配合物 41 44. 41 44 在晶态由 于 RIR 效应表现出 AIPE 性质. 基于 $41 \sim 44$ 的 AIPE 活 性, 用聚丙烯酰胺与聚苯乙烯包覆制备的 41 44 的聚合 物纳米粒子, 实现了肿瘤细胞的靶向成像. Lalinde 等 ${ }^{[66]}$ 报道了含氟代苯基吡啶配体的铂(II)配合物 45, 该配合 物的平面结构使其在聚集态产生 $\pi \cdots \pi$ 堆积, 使得分子 间和分子内运动受限，从而显示 AIE 特性. 而且，45 受 力后发生可逆的晶态到非晶态的相变, 使其具有可逆的 MCL 特性. 2018 年, Ionescu 等 ${ }^{[67]}$ 报道了铂(II)配合物 46a 和铂(IV)配合物 $46 \mathrm{~b}$. 相对于不含氟的类似物, 氟的引 入使配合物 46 具有更长的磷光寿命. 2012 年, Lalinde

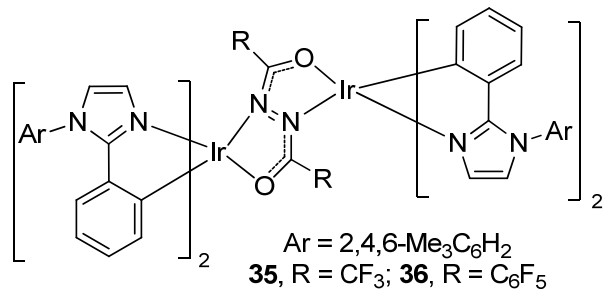

$35, \mathrm{R}=\mathrm{CF}_{3} ; 36, \mathrm{R}=\mathrm{C}_{6} \mathrm{~F}_{5}$
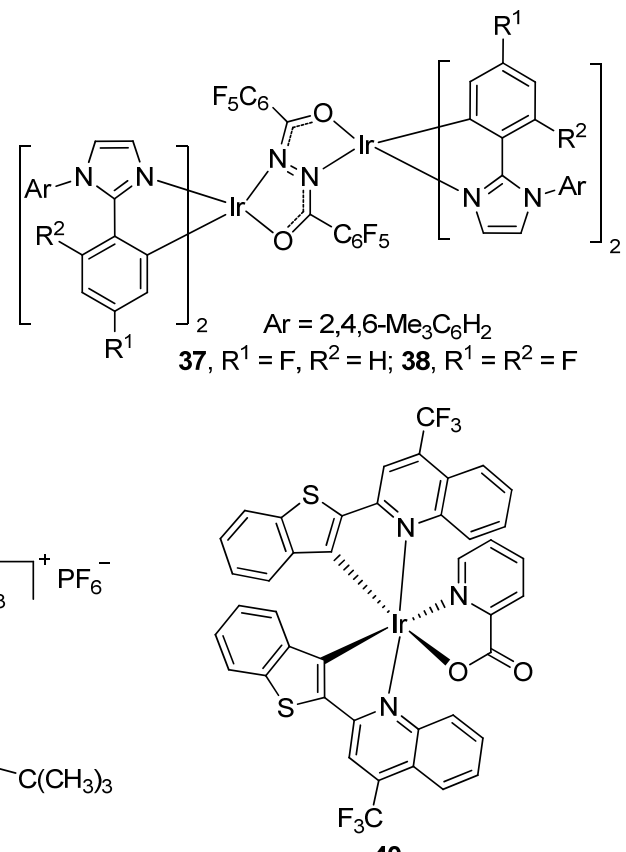

39

40 
表 2 含氟 AIE 铂配合物的光物理性质和潜在应用

Table 2 Photophysical properties and potential applications of fluorinated platinum complexes

\begin{tabular}{|c|c|c|c|c|c|c|}
\hline 化合物 & 吸收峰 ${ }^{a} / \mathrm{nm}$ & 发射峰 ${ }^{b} / \mathrm{nm}$ & 发光量子效率 ${ }^{b} / \%$ & 发光寿命 ${ }^{b}$ & 潜在应用 & 文献 \\
\hline 41 & $261,301,321,354,386,433$ & - & - & - & 细胞成像 & {$[65]$} \\
\hline 42 & $258,321,354,383,428$ & - & - & - & 细胞成像 & {$[65]$} \\
\hline 43 & $262,325,351,386,428$ & - & - & - & 细胞成像 & {$[65]$} \\
\hline 44 & $263,306,360,396,448$ & - & - & - & 细胞成像 & {$[65]$} \\
\hline 45 & $265,286,315,330,406$ & $486,518,575$ & 29 & - & - & [66] \\
\hline $46 a$ & $281,313,343,480$ & 600 & 35 & $2.88 \mu \mathrm{s}$ & - & {$[67]$} \\
\hline $46 b$ & $300,320,389,465$ & 521,621 & 10 & $\begin{array}{l}5.9 \mathrm{~ns}(521 \mathrm{~nm}) \\
488.51 \mathrm{~ns}(621 \mathrm{~nm})\end{array}$ & - & {$[67]$} \\
\hline 47 & $247,275,291,304,329,353$ & $\begin{array}{l}480,515,585\left(\lambda_{\text {ex }}: 400\right) \\
595\left(\lambda_{\text {ex }}: 445 \sim 475\right) \\
506,540,580\left(\lambda_{\text {ex }}: 400,77 \mathrm{~K}\right)\end{array}$ & - & $\begin{array}{l}13.2 \mu \mathrm{s}(480 \mathrm{~nm}) \\
16.5 \mu \mathrm{s}(515 \mathrm{~nm}) \\
157 \mu \mathrm{s}(506 \mathrm{~nm})\end{array}$ & 细胞成像 & {$[68]$} \\
\hline $48 \mathbf{a}$ & $\begin{array}{l}252,265,282,304,347 \\
394,465\end{array}$ & $\begin{array}{l}565,625\left(\lambda_{\mathrm{ex}}: 400 \sim 470\right) \\
520,555\left(\lambda_{\mathrm{ex}}: 400,77 \mathrm{~K}\right) \\
555,605\left(\lambda_{\mathrm{ex}}: 440,77 \mathrm{~K}\right)\end{array}$ & - & $\begin{array}{l}11.1 \mu \mathrm{s}(565 \mathrm{~nm}) \\
488 \mu \mathrm{s}(625 \mathrm{~nm}) \\
41.7 \mu \mathrm{s}(520 \mathrm{~nm}) \\
42.1 \mu \mathrm{s}(555 \mathrm{~nm}) \\
47.9 \mu \mathrm{s}(605 \mathrm{~nm})\end{array}$ & 细胞成像 & {$[68]$} \\
\hline $48 b$ & $\begin{array}{l}236,264,255,288,306 \\
333,356,399\end{array}$ & $\begin{array}{l}609\left(\lambda_{\mathrm{ex}}: 400 \sim 470\right) \\
510,575,610\left(\lambda_{\mathrm{ex}}: 380,77 \mathrm{~K}\right) \\
555,575,610\left(\lambda_{\mathrm{ex}}: 440,77 \mathrm{~K}\right)\end{array}$ & - & $\begin{array}{l}12.8 \mu \mathrm{s}(609 \mathrm{~nm}) \\
167 \mu \mathrm{s}(510 \mathrm{~nm}) \\
112 \mu \mathrm{s}(575 \mathrm{~nm}) \\
65.2 \mu \mathrm{s}(610 \mathrm{~nm})\end{array}$ & 细胞成像 & {$[68]$} \\
\hline $48 \mathrm{c}$ & $\begin{array}{l}237,252,280,302,333 \\
362,406\end{array}$ & $\begin{array}{l}600\left(\lambda_{\mathrm{ex}}: 400 \sim 470\right) \\
481,500,523,538\left(\lambda_{\mathrm{ex}}: 380,77 \mathrm{~K}\right) \\
481,500,523,538,613\left(\lambda_{\mathrm{ex}}: 440,\right. \\
77 \mathrm{~K})\end{array}$ & - & $\begin{array}{l}13.9 \mu \mathrm{s}(600 \mathrm{~nm}) \\
76.6 \mu \mathrm{s}(500 \mathrm{~nm}) \\
42.8 \mu \mathrm{s}(613 \mathrm{~nm})\end{array}$ & 细胞成像 & {$[68]$} \\
\hline $49 a$ & $243,261,290,309,337,370$ & $\begin{array}{l}496,595\left(\lambda_{\mathrm{ex}}: 400\right) \\
595\left(\lambda_{\mathrm{ex}}: 450 \sim 480\right) \\
507,544,586\left(\lambda_{\mathrm{ex}}: 400,77 \mathrm{~K}\right)\end{array}$ & - & $\begin{array}{l}9.0 \mu \mathrm{s}(495 \mathrm{~nm}) \\
14.5 \mu \mathrm{s}(595 \mathrm{~nm}) \\
186 \mu \mathrm{s}(507 \mathrm{~nm})\end{array}$ & 细胞成像 & {$[68]$} \\
\hline $49 \mathrm{~b}$ & $243,261,290,309,337,370$ & $\begin{array}{l}545\left(\lambda_{\mathrm{ex}}: 400\right) \\
508,532,572\left(\lambda_{\mathrm{ex}}: 400,77 \mathrm{~K}\right) \\
570\left(\lambda_{\mathrm{ex}}: 470\right)\end{array}$ & - & $\begin{array}{l}9.1 \mu \mathrm{s}(545 \mathrm{~nm}) \\
202.2 \mu \mathrm{s}(532 \mathrm{~nm}) \\
20.1 \mu \mathrm{s}(570 \mathrm{~nm})\end{array}$ & 细胞成像 & {$[68]$} \\
\hline 50 & - & 573 & - & - & - & [69] \\
\hline 54 & 280,360 & 600 & 65 & $404 \mathrm{~ns}$ & - & {$[71]$} \\
\hline 55 & 365,420 & 495 & - & - & - & {$[72]$} \\
\hline
\end{tabular}

${ }^{a}$ Solution; ${ }^{b}$ Solid; - : Unreported.<smiles></smiles>

41<smiles></smiles><smiles></smiles>

42<smiles></smiles>

43<smiles></smiles>

44

\section{m}


等 ${ }^{[68]}$ 报道了含五氟苯基配体的铂(II)配合物 $47 \sim 49$, 配 合物的平面结构使其产生 $\pi \cdots \pi$ 堆积, 导致其在聚集态 发光, 可用于肿瘤细胞成像领域.<smiles></smiles>

47

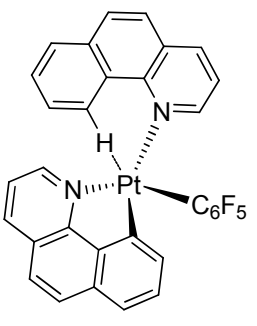

49a

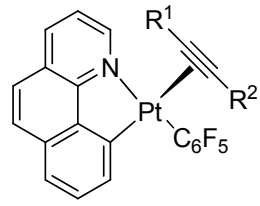

48a, $R^{1}, R^{2}=P h$

48b, $R^{1}=H, R^{2}=P h$

48c, $\mathrm{R}^{1}=\mathrm{H}, \mathrm{R}^{2}={ }^{t} \mathrm{Bu}$

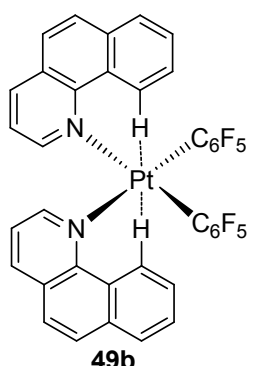

$49 b$
2018 年, Shiotsuka 等 ${ }^{\left[{ }^{[9]}\right.}$ 合成了铂配合物 50, 由于其 含有的芳基炔基配体有利于固态下配合物分子间的紧 密堆积, 限制分子内振动与旋转, 在固态下发光较强, 而其脱硅基保护的端炔类似物, 不具有 AIE 特性. 支志 明等 ${ }^{[70]}$ 报道了分别含有三齿 $\mathrm{C}^{\wedge} \mathrm{N}^{\wedge} \mathrm{N}$ 和 $\mathrm{N}^{\wedge} \mathrm{C}^{\wedge} \mathrm{N}$ 螯合配体 的铂配合物 51 和 52 , 并借助不同比例的水/甲醇溶液研 究了聚集态对 51 和 52 发光性质的影响. 随着含水量的 增加, Pt ‥Pt 相互作用也促进了聚集体的形成, 51 和 52 的发射峰逐渐红移, 发光强度也逐渐增加. 2017 年, De Cola 等 ${ }^{[71]}$ 报道了两个含有 $\mathrm{N}^{\wedge} \mathrm{C}^{\wedge} \mathrm{N}$ 螯合配体的铂配合物 53 和 54.53 和 54 不具有典型的 AIE 性质, 但具有聚集 诱导电化学发光 (Aggregation-Induced Electrochemiluminescence, AIECL)特性. 化合物 $\mathbf{5 4}$ 在二氯甲烷/水混合 体系和固态均具有电化学发光(ECL)性能, 而化合物 $\mathbf{5 3}$ 只在固态时在机械应力作用下显示 ECL 特性. 2019 年, 李维军等 ${ }^{[72]}$ 合成了含有三齿 $\mathrm{N}^{\wedge} \mathrm{C}^{\wedge} \mathrm{N}$ 螯合配体的铂配合 物 55 . 从 55 的晶体堆积结构推知, 55 的 AIE 特性可以 归因于 RIR 效应. 另外, 在光激发下 $\mathbf{5 5}$ 发生自敏化氧化 反应, 其中一个咪唑环从碳碳双键处开环, 而开环后形 成的铂配合物不具有 AIE 特性.

\section{3 含氟金配合物}

金(I)配合物的合成和发光性质被广泛研究与报道. 由于金原子间的亲金相互作用(aurophilic interaction), 金配合物在聚集态下容易形成超分子聚集体, 从而具有 AIE 特性 ${ }^{[73]}$. 目前报道的含氟 AIE 金配合物有单核、双 核、三核及四核的结构. 在这些配合物中, 金都是一价, 大都含有吸电子的五氟苯基异腈配体. 五氟苯基的引
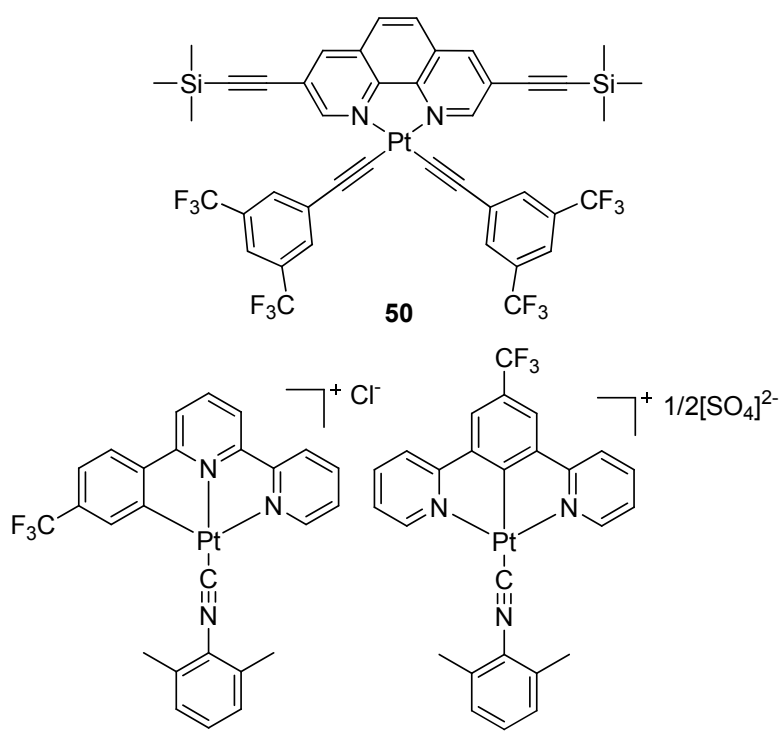

51

52

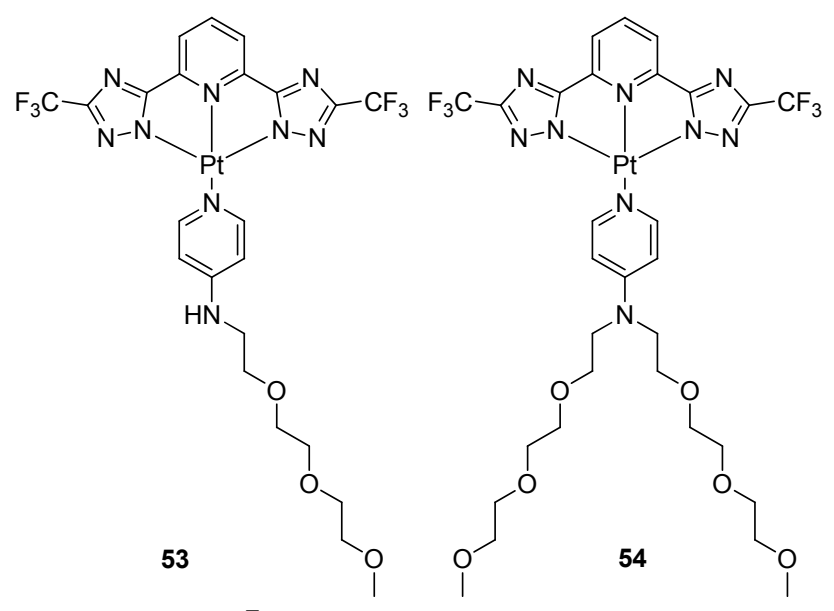<smiles></smiles>

入, 使这些金配合物分子间具有 $\mathrm{C}-\mathrm{H} \cdots \mathrm{F}$ 和 $\pi \cdots \pi$ 相互 作用，连同金原子间可能的亲金相互作用，使金配合物 具有显著的 AIE、MCL 及热致发光变色等特性. 在这一 研究方向, 刘盛华等课题组做了较系统的工作. 表 3 汇 总了含氟 AIE 金配合物的光物理性质及其潜在应用.

2013 年, 刘盛华、余广鳌等 ${ }^{[74]}$ 报道了具有 AIE 和热 致发光变色特性的单核金配合物 $\mathbf{5 6}$, 其苂光随温度升 高表现出可逆的从蓝到黄绿色的变化(图 6). 2015 年, 刘 盛华等 ${ }^{\left[{ }^{[5]}\right.}$ 报道了由不同长度烷基链连接的咔唑基单核 金配合物 57, 该配合物具有 AIE 和可逆的 MCL 特性. 2016 年, 刘盛华等 ${ }^{[76]}$ 报道了具有不同芴基的单核金配 
表 3 含氟金配合物的光物理性质和潜在应用

Table 3 Photophysical properties and potential applications of fluorinated gold complexes

\begin{tabular}{|c|c|c|c|c|c|c|}
\hline 化合物 & 吸收峰 ${ }^{a} / \mathrm{nm}$ & 发射峰 $b / \mathrm{nm}$ & 发光量子效率 ${ }^{b} / \%$ & 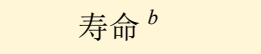 & 潜在应用 & 文献 \\
\hline 56 & - & 407,428 & - & - & - & [74] \\
\hline $57(n=2)$ & $280,328,342$ & - & - & - & - & {$[75]$} \\
\hline $57(n=5)$ & - & 554,596 & - & - & - & {$[75]$} \\
\hline 60 & - & 467,498 & - & - & - & {$[76]$} \\
\hline $61 \mathrm{a}$ & - & 513,552 & - & - & - & [77] \\
\hline $61 b$ & - & 530 & - & - & - & {$[77]$} \\
\hline 62 & 336 & $462,550,598$ & 22.5 & $86.84 \mathrm{~ms}(550 \mathrm{~nm})$ & - & {$[78]$} \\
\hline 63 & - & 454 & 21.4 & $1.53 \mu \mathrm{s}$ & - & [79] \\
\hline 64 & - & 453,560 & - & $13.72 \mu \mathrm{s}(560 \mathrm{~nm})$ & - & {$[80]$} \\
\hline 65 & - & 526 & - & $30.79 \mu \mathrm{s}$ & - & {$[80]$} \\
\hline 66 & - & 512 & - & $4.20 \mu \mathrm{s}$ & - & {$[80]$} \\
\hline 67 & 254 & 556 & 3.7 & - & - & {$[81]$} \\
\hline 68 & 255 & 557 & 6.3 & - & - & [81] \\
\hline 69 & 239 & 419 & 27.7 & - & - & [81] \\
\hline 70 & 306 & 627 & - & - & - & [81] \\
\hline 71 & 238,325 & $512,551,596$ & - & - & - & [81] \\
\hline $72(n=2)$ & - & 485 & - & $0.77 \mu \mathrm{s}$ & - & [82] \\
\hline 73 & $266,280,352$ & - & 65.42 & - & - & [83-84] \\
\hline 74 & - & 499 & 49.46 & - & - & {$[84]$} \\
\hline 75 & - & 389,501 & 5.42 & - & - & [84] \\
\hline 76 & $304,324,338$ & 490,523 & 40.51 & - & - & {$[85]$} \\
\hline 77 & - & 496 & - & - & - & [86] \\
\hline 78 & - & 516 & - & - & - & {$[86]$} \\
\hline 79 & - & 430 & - & - & - & [87] \\
\hline 82 & 270 & $572^{\mathrm{a}}$ & - & - & - & [88] \\
\hline $84(n=5)$ & - & 505,540 & 5 & $\begin{array}{l}18.37 \mu \mathrm{s}(505 \mathrm{~nm}) \\
16.00 \mu \mathrm{s}(540 \mathrm{~nm})\end{array}$ & & [89] \\
\hline $84(n=6)$ & - & 500,540 & 4 & $\begin{array}{l}22.43 \mu \mathrm{s}(500 \mathrm{~nm}) \\
22.72 \mu \mathrm{s}(540 \mathrm{~nm})\end{array}$ & & [89] \\
\hline $84(n=7)$ & - & 505,540 & 4 & $\begin{array}{l}15.97 \mu \mathrm{s}(505 \mathrm{~nm}) \\
16.72 \mu \mathrm{s}(540 \mathrm{~nm})\end{array}$ & $\begin{array}{l}\text { 自擦除复写纸 } \\
\text { 矛性防伪王碳 }\end{array}$ & [89] \\
\hline $84(n=8)$ & - & 500,535 & 68 & $\begin{array}{l}201.68 \mu \mathrm{s}(500 \mathrm{~nm}) \\
184.05 \mu \mathrm{s}(535 \mathrm{~nm})\end{array}$ & $\begin{array}{l}\text { 禿性邚伪 } \\
\text { 复印纸 }\end{array}$ & [89] \\
\hline $84(n=9)$ & - & 500,535 & 64 & $\begin{array}{l}206.00 \mu \mathrm{s}(500 \mathrm{~nm}) \\
201.48 \mu \mathrm{s}(535 \mathrm{~nm})\end{array}$ & & [89] \\
\hline $84(n=10)$ & - & 500,535 & 16 & $\begin{array}{l}127.47 \mu \mathrm{s}(500 \mathrm{~nm}) \\
124.61 \mu \mathrm{s}(535 \mathrm{~nm})\end{array}$ & & [89] \\
\hline 85 & - & 470 & - & - & 生物传感 & {$[90]$} \\
\hline $86(R, R)$ & - & $504^{a}, 500$ & 15 & $12 \mathrm{~ms}$ & - & [91] \\
\hline $86(S, S)$ & - & $504^{a}, 500$ & 16 & $8 \mathrm{~ms}$ & - & [91] \\
\hline 87 & - & 527 & - & $0.87 \mu \mathrm{s}$ & - & [92] \\
\hline 88 & - & 500 & 15.1 & - & - & [93] \\
\hline 89 & & 498 & 8.1 & - & - & [93] \\
\hline 90 & & 498 & 8.3 & - & - & [93] \\
\hline 91 & - & 389 & 5.8 & - & - & [84] \\
\hline 92 & - & $376,452,471$ & 10.63 & - & - & {$[84]$} \\
\hline
\end{tabular}

${ }^{a}$ Solution; ${ }^{b}$ Solid; - : Unreported.

合物 58 60. 这些金配合物均表现出明显的 AIE 特征. 相对于 58 和 59 , 芴基上不具有烷基链的 60 表现出可逆 的 MCL 特性, 研磨后菼光颜色由绿变为淡黄.
2016 年, 刘盛华、余广鳌等 ${ }^{[77]}$ 合成了一系列不同长 度 $N$-烷基取代的咔唑基单核金配合物 61. 这些配合物 均具有明显的 AIE 特征, 且 $61 \mathrm{a}$ 和 $61 \mathrm{~b}$ 还具有可逆的 
MCL 特性, 研磨后荧光颜色由绿变为黄绿. 2017 年, 刘 盛华等 ${ }^{[78]}$ 报道了咔唑基单核金配合物 62.62 具有明显 的 AIE、可逆的 MCL 和蒸气致发光变色特性, 研磨后 其发光从黄色变为黄绿色; 用二氯甲烷溶剂熏蒸后, 其 发光恢复到黄色, 且 62 还具有室温磷光特性. 同年, 袁 望章等 ${ }^{[79]}$ 合成了含四苯基乙烯的、具有 $\mathrm{AIPE}$ 及 $\mathrm{MCL}$ 特性的金配合物 63 , 其晶体发光在研磨后表现出可逆 的从蓝(454 nm)到绿(500 nm)的变化. 2018 年, 刘盛华等 ${ }^{[80]}$ 报道了单核金配合物 $64 \sim 66$. 这些金配合物, 均表现 出明显的 AIE 特征, 且 64 和 65 还具有可逆的 MCL 性
质，原因是分子间 $\mathrm{C}-\mathrm{H} \cdots \mathrm{F}$ 键或 $\pi-\pi$ 作用的变化以及亲 金相互作用. 2018 年，刘盛华等 ${ }^{[81]}$ 报道了单核金配合物 67 71. 67 71 均具有明显的 AIE 和可逆的 MCL 特性. 含氟 AIE 双核金配合物也报道较多. 2014 年，刘盛 华、余广鳌等 ${ }^{[82]}$ 合成了不同长度醚氧桥联二异腈配合物 72, 其均表现出 AIE 和 MCL 行为. 光谱学、发光量子 效率和 X射线粉末衍射等结果证明，分子间的 $\mathrm{C}-\mathrm{H} \cdots \mathrm{F}$ 和 $\pi-\pi$ 相互作用的变化以及亲金作用是其具有 AIE 和 $\mathrm{MCL}$ 性质的关键原因. 随后，刘盛华、余广鳌等 ${ }^{[83-84]}$ 报 道了三种芴基配合物 73 75. 73 75 的发光颜色随芴单
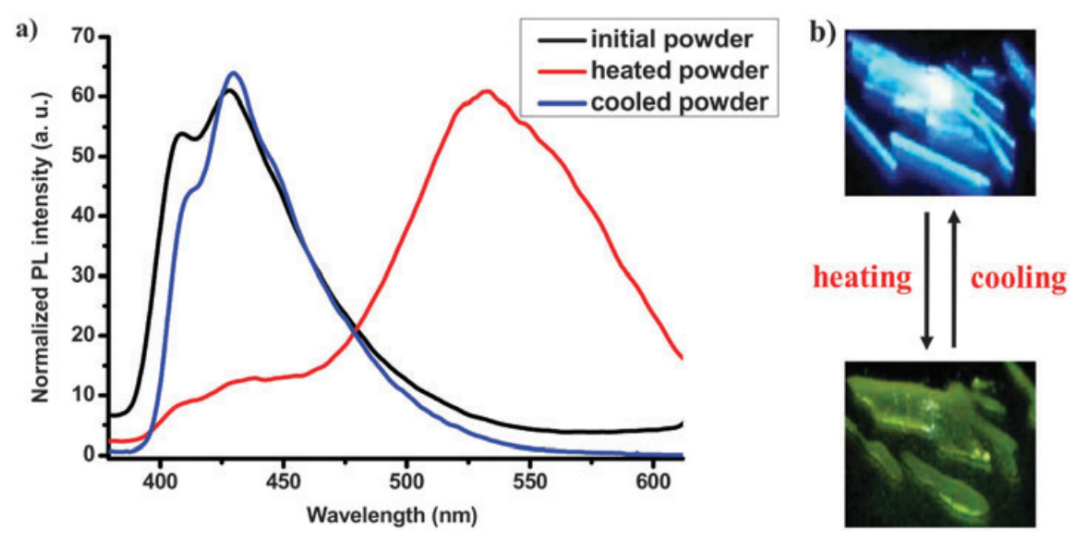

图 656 的(粉末、加热和冷却后)归一化 PL 光谱(a)和在 $365 \mathrm{~nm}$ 紫外光下加热前后的照片(b) ${ }^{[74]}$

Figure 6 Normalized PL spectra (initial, heated and cooled) (a) and images before and after heating under $365 \mathrm{~nm}$ UV illumination (b) of $\mathbf{5 6}^{[74]}$

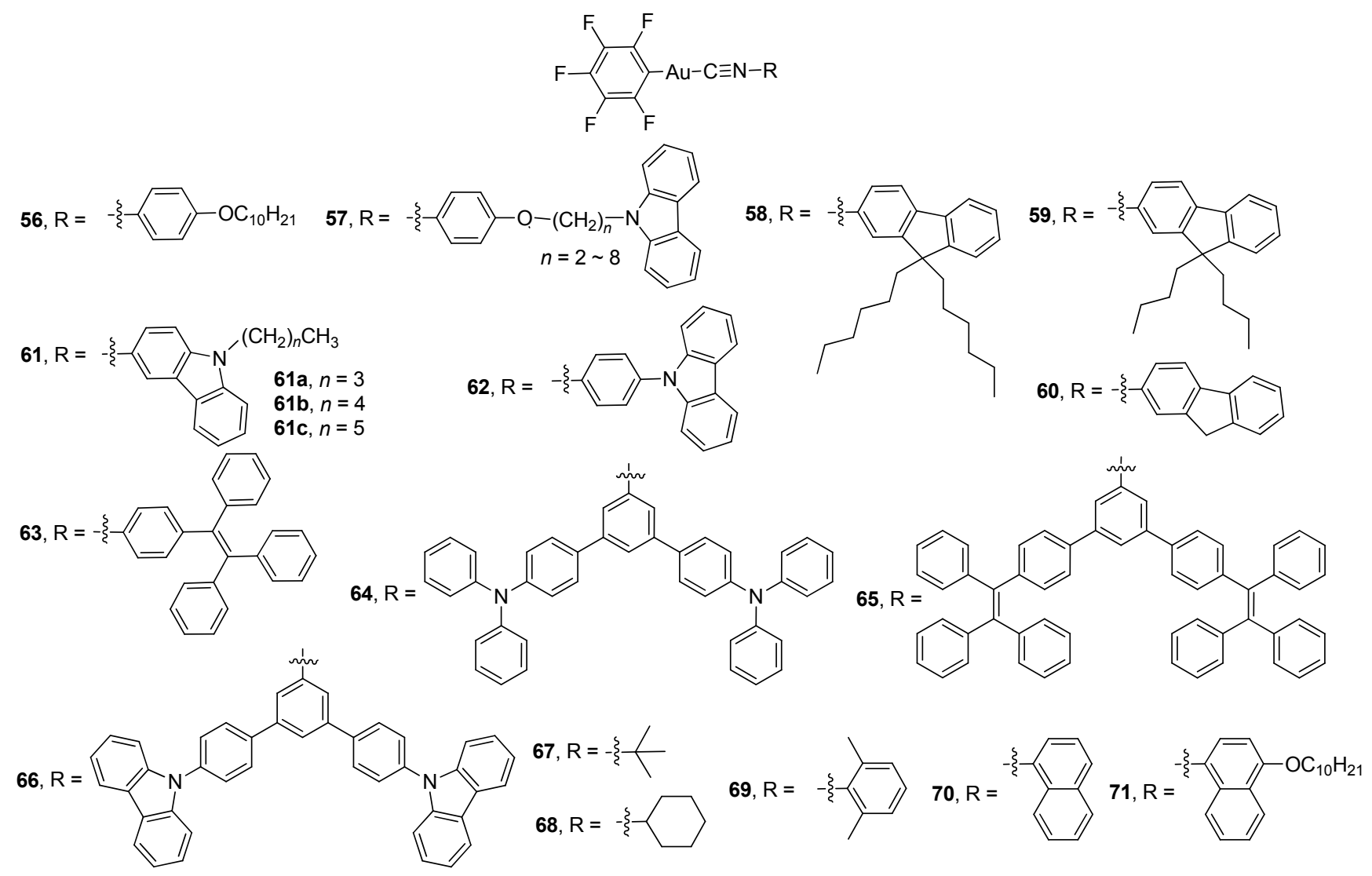


元的不同而变化, 含有三个芴单元的配合物 73 在固态 和薄膜态都发明亮的白色苂光, 且发光量子效率较高. 由于氟原子引入而引起的分子间 $\mathrm{C}-\mathrm{H} \cdots \mathrm{F}$ 氢键及亲金 相互作用导致其聚集态结构重排, 73 的荧光随聚集程度 而发生变化. 在 $N, N$-二甲基甲酰胺(DMF)/水混合体系 中, 当含水量为 0 时溶液不发光, 随着含水量增大, 73 逐渐聚集成颗粒，并在含水量小于 $30 \%$ (体积分数)时发 蓝绿光，当含水量大于 $30 \%$ 时则发白光.

2015 年，刘盛华、余广鳌等报道了配合物 $\mathbf{7 6}^{[85]}, \mathbf{7 7}$, $78^{[86]}, 79 \sim 81^{[87]}$. 这些金配合物, 均表现出明显的 AIE 特性. 而且, 76 具有结晶诱导发光增强(crystallizationinduced emission enhancement, CIEE)及可逆的 MCL 性 质, 79 具有可逆的 MCL 性质, 80 则具有力致发光增强性 质, 而 81 无 MCL 性质. X射线衍射结果表明, 晶态和非 晶态之间的形貌转变是导致其具有 MCL 或 CIEE 特性 的主要原因, 这种形貌转变是由于五氟苯基使分子间形
成 $\mathrm{C}-\mathrm{H} \cdots \mathrm{F}$ 氢键引起的.

2015 年, 刘盛华等 ${ }^{[88}$ 合成了配合物 82. 82 在乙腈/ 水体系中表现出典型的 AIE 特性, 发光随含水量的增加 而逐渐增强, 且 82 在乙腈/水体系中与 $\mathrm{Hg}^{2+}$ 发生配位反 应引起苂光猝灭，使 83 可以用于快速检出体系中的 $\mathrm{Hg}^{2+}$ 离子. 2016 年, 刘盛华、余广鳌等 ${ }^{[77]}$ 合成了一系列 不同 $N$-烷基链长度的咔唑基配合物 83. 83a 83c 均表 现出明显的 AIE 特性, 其中 $83 a$ 还表现出可转换的 MCL 行为, 研磨后苂光颜色从蓝色变为绿色. $83 \mathrm{~b}$ 和 $83 \mathrm{c}$ 的固 态苂光可以通过机械力刺激在弱苂光和强苂光之间转 换, 这种转换是不可逆的. 刘盛华、唐本忠等 ${ }^{[89]}$ 合成了 一系列金配合物 $84(n=5 \sim 10)$, 这些配合物在固态发出 绿色磷光，其发射峰在 $500 \sim 535 \mathrm{~nm}$ ，在室温下其发光 寿命为 $16 \sim 206 \mu \mathrm{s}$, 发光量子效率为 $4 \% \sim 68 \%$, 烷基链 的长度对 84 的固态发光有明显的影响，其中 $n=8,9$ 时, 配合物的发光量子效率分别为 $68 \%$ 和 $64 \%$, 发光量子效<smiles></smiles>

72, $R=$<smiles>CCOC(C)(C)COc1ccc(C(C)(C)C)cc1</smiles>
73, $R=$

75, $\mathrm{R}$<smiles>CC(C)(C)c1ccc(OCCCCCCC2(CCCCCCOc3ccc(C(F)(F)F)cc3)C=CC3=CC=C(Cc4ccc5c(c4)-c4ccc3cc4C5)c3ccccc32)cc1</smiles><smiles>CCCC1(CC)c2cc(-c3ccc4c(c3)Cc3ccccc3-4)ccc2-c2ccc(-c3ccc4c(c3)Cc3ccccc3-4)cc21</smiles>

$74, R=$<smiles>CC(C)(C)c1ccc(OCCCCCCCC2(CCCCCCOc3ccc(C(F)(F)F)cc3)c3ccccc3-c3ccccc32)cc1</smiles>

76, $R=$<smiles>[Y]c1ccc2c(c1)C(CCCCCC)(CCCCCC)c1ccccc1-2</smiles>

77, $\mathrm{R}=$<smiles>Cc1ccc(Oc2ccc(-c3ccc(Oc4ccc(C(C)(C)C)cc4)cc3)cc2)cc1</smiles>

79, $R$<smiles>Cc1ccc(Oc2ccccc2Oc2ccc(I)cc2)cc1</smiles>

$80, R=$<smiles>Oc1ccccc1</smiles><smiles>Cc1ccc(Oc2cccc(I)c2)cc1</smiles>

$83, R=$<smiles>CCCCNc1ccc(C)cc1</smiles><smiles></smiles>

$83 \mathbf{a}, n=3$

$83 \mathrm{c}, n=5$<smiles>[Y]c1ccc(Oc2ccc(Oc3ccc([Y]([Y])([H])Br)cc3)cc2)cc1</smiles>

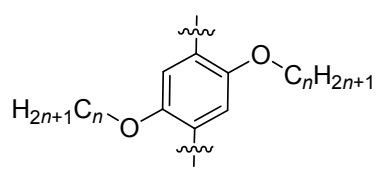

$n=5,6,7,8,9,10$ 
率高的原因可能是较容易结晶. 同时 84 具有自恢复的 MCL 特性, 且自恢复速度随着烷基链长度的增加而加 快, 其可逆 MCL 特性在自擦除复写纸和柔性防伪无碳 复印纸材料方面具有应用潜力(图 7).

手性的 AIE 含氟有机金属配合物，最近也有报道. 刘盛华、唐本忠等 ${ }^{[90]}$ 报道了手性的双核金配合物 85, 两 个对映异构体在聚集态( THF $/ \mathrm{H}_{2} \mathrm{O}$ 体系)随时间的延长都 形成螺旋结构组装体, 虽然该组装体不具有明显的圆偏 振发光(CPL, circularly polarized luminescence)性质, 但 可作为手性模板与非手性的 AIE 分子[四苯基乙烯、 9,10-二苯乙炔基葸、2,3,5,6-四(4-甲氧基苯基)吡嗪]共组 装诱导产生 CPL 发光, 该方法为获得具有 CPL 特性的
发光材料提供了一种简便易行的策略. 最近，唐本忠 等 ${ }^{[91]}$ 报道了手性的双核金配合物 86. 与绝大多数 AIE 材料不同，这两个对映体的粉末不发光，但粉末的纳米 级维晶在机械力刺激下 (刮刀即可)借助分子间的亲金相 互作用及 $\mathrm{C}-\mathrm{H} \cdots \mathrm{F}$ 和 $\pi-\pi$ 相互作用组装成高度有序的微 晶而发光. 而且, 在晶相保持完全不变的情况下, 通过 巧妙调控分子层间滑动及更细微的压缩就可实现磷光 开到关的切换. 两种异构体的粉末研磨后也具有 CPL 性能，其发光不对称因子达 $4 \times 10^{-3}$.

2018 年, 刘盛华等 ${ }^{[92]}$ 报道了一个含有联吡啶桥联 配体的双核金配合物 87.87 具有 AIPE 以及可逆的 MCL 特性, 研磨后磷光由绿色变为亮黄色.
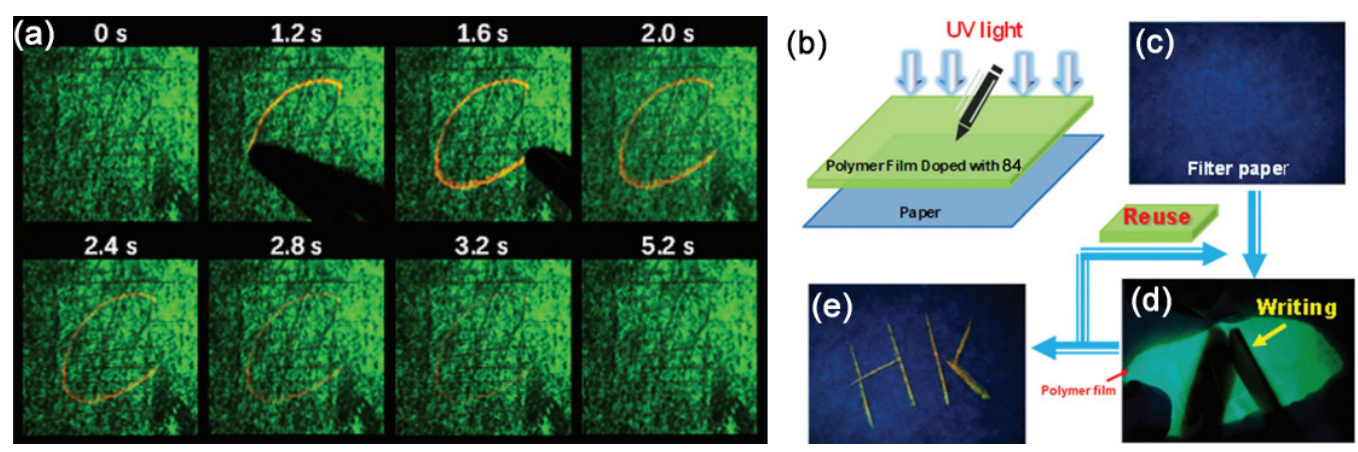

图 7 (a)含配合物 84 的可擦写纸张上自擦除过程的演示、(b)配合物 84 用于无碳复写纸系统的示意图、(c)含配合物 84 的空白基 板(滤纸)的苂光图像、(d)在滤纸上贴上一层薄膜，用镊子在薄膜上写上字母“HK”及(e)滤纸揭膜后的苂光图像 ${ }^{[89]}$

Figure 7 (a) Demonstration of the self-erasing process on rewritable paper of complex 84, (b) schematic representation of the system used as carbonless copy paper of complex 84, (c) fluorescence image of the blank substrate (filter paper) of complex 84, (d) A thin-film was put on the top of the filter paper, and the letters "HK" were written on the thin-film with tweezers and (e) fluorescence image of filter paper after uncovering the thin-film ${ }^{[89]}$

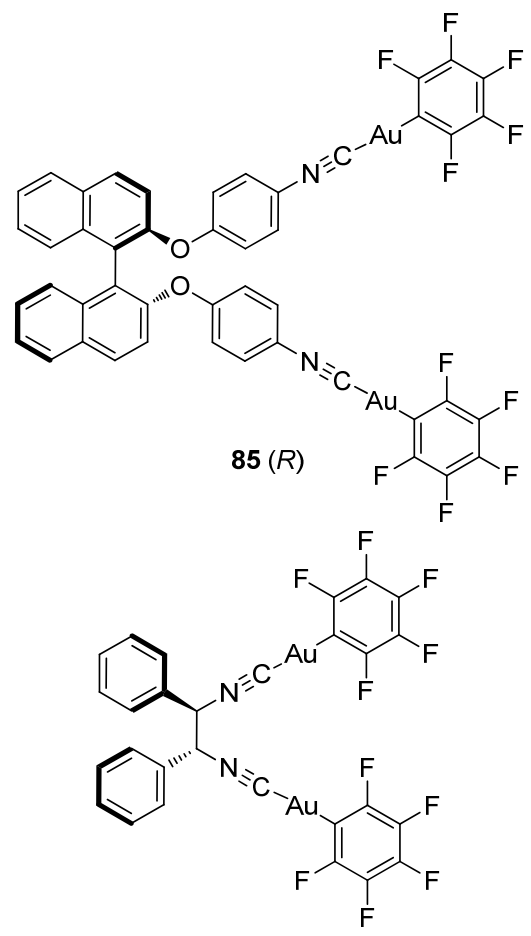

$86(R, R)$

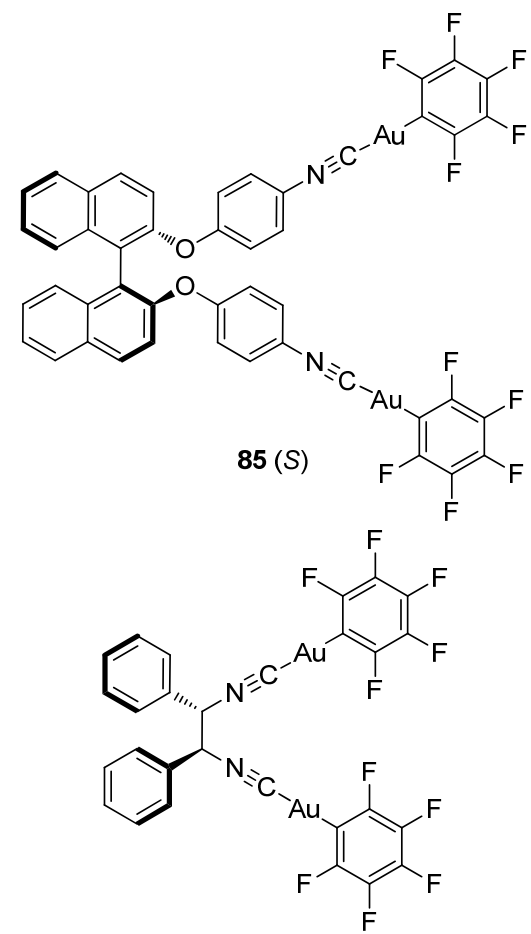

$86(S, S)$ 


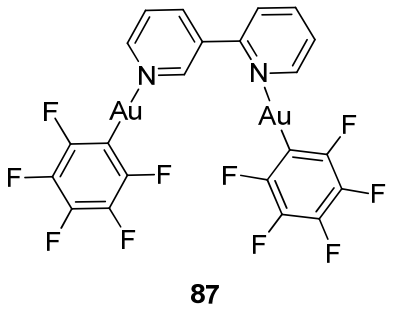

三核及四核的金配合物也有一些报道. 2015 年, 刘 盛华、余广鳌等报道了三核金配合物 88 $\mathbf{9 0}^{[93]}$. 这些配 合物均表现出明显的 AIE 和可逆的 MCL 特性. 晶体结 构分析表明, 氟原子的引入导致分子间 $\mathrm{C}-\mathrm{H} \cdots \mathrm{F}$ 氢键 与 $\pi-\pi$ 相互作用的变化, 加上亲金作用, 使其具有 AIE 及 MCL 特性. 2015 年, 刘盛华、余广鳌等 ${ }^{[84]}$ 合成了两种 芴基四核金配合物 91 和 92, 两者均表现出典型的 AIE 特性, 发光颜色随葋单元的不同而变化, 且 92 在薄膜和 固态下发白色苂光.<smiles>[R]c1cccc([R])c1[R]</smiles>

88<smiles>[R]c1ccc([R])c([R])c1</smiles>

89<smiles>[R]c1ccc([R])cc1</smiles>

90

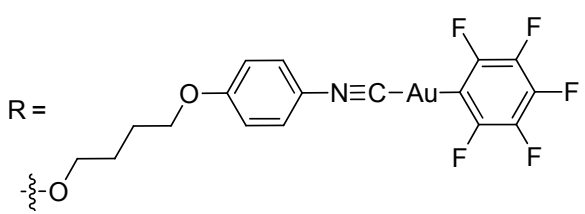<smiles>[R]C1c2ccccc2-c2ccc(-c3ccc4c(c3)-c3ccccc3C4([R])[R])cc21</smiles><smiles></smiles><smiles></smiles>

这些金配合物由于 RIR 效应、亲金相互作用及 C$\mathrm{H} \cdots \mathrm{F}$ 和 $\pi-\pi$ 相互作用, 增强了分子间作用力, 使化合物 具有 AIE 特性. 五氟苯基配体的引入也使这些金配合物 具有较高的固态发光量子效率及对压力、温度、溶剂蒸 气等的刺激响应性, 这些性质对于设计合成新的刺激响 应材料及其应用很有意义.

\section{4 含氟其它金属的配合物}

除了上述铱、铂、金的配合物, 还有少量其它金属
的含氟 AIE 有机金属配合物报道(表 4 ).

最近, 朱红平等 ${ }^{[94]}$ 合成了铝杂环戊二烯配合物 93 和 94, 含氟配体的引入使其在固态时发生分子内旋转 受限, 发出明亮的黄色荧光.

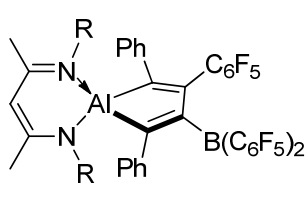

93, $\mathrm{R}=2,6-{ }^{i} \mathrm{Pr}_{2} \mathrm{C}_{6} \mathrm{H}_{3}$

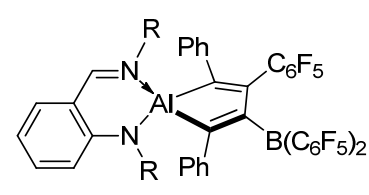

94, $\mathrm{R}=2,6-{ }^{-} \mathrm{Pr}_{2} \mathrm{C}_{6} \mathrm{H}_{3}$
Braddock-Wilking 等 ${ }^{[95]}$ 合成了锗杂环伐二烯化合物 95 97. 95 97 的外围取代基在聚集态下的 RIR 效应, 使其具有 AIE 特性. 相对于不含氟的类似物, 这些配合 物的固态发射波长发生少许蓝移, 斯托克斯位移也变 小. 95 97 的薄膜暴露于丙酮蒸气中, 发生荧光猝灭, 而当除去丙酮蒸气，苂光又恢复. 因此，95 97 能用作 挥发性有机化合物如丙酮的苂光传感器.
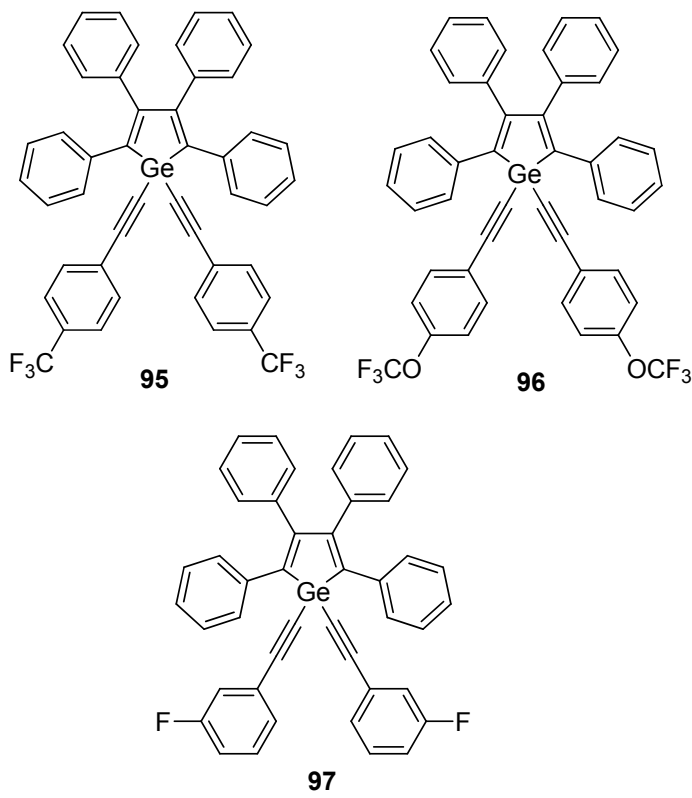

\section{5 结论及展望}

目前报道的具有 AIE 性质的、含有氟代配体的有机 金属配合物，主要是铱、铂、金的配合物，其含氟配体 主要是苯基吡啶(铱、铂)、苯基吡唑(铱)、苯基咪唑(铱)、 五氟苯基(铂、金)以及一些其他配体. 配体中的氟原子, 往往具有特殊的效应，通过氢键等方式影响分子间相互 作用及聚集态堆积结构, 使这些含氟配合物具有与其非 氟代类似物不同的发光性质。这些含氟 AIE 有机金属配 合物, 不但扩展了 AIE 化合物的研究范围, 还在生物医 学、光电材料、信息存储及传感等领域具有潜在的应用.

针对目前含氟 AIE 有机金属配合物数量和种类较 
表 4 含氟铝、锗配合物的光物理性质和潜在应用

Table 4 Photophysical properties and potential applications of fluorinated aluminum and germanium complexes

\begin{tabular}{|c|c|c|c|c|c|c|}
\hline 化合物 & 吸收峰 ${ }^{a} / \mathrm{nm}$ & 发射峰 ${ }^{b} / \mathrm{nm}$ & 发光量子效率 ${ }^{b} / \%$ & 发光寿命 ${ }^{b} / \mathrm{ns}$ & 潜在应用 & 文献 \\
\hline 93 & 337,365 & 565 & 9.3 & 13.7 & - & {$[94]$} \\
\hline 94 & 337,366 & 566 & 8.6 & 4.3 & - & [94] \\
\hline 95 & 369 & 486 & - & & 化学传感 & [95] \\
\hline 96 & 365 & 485 & - & & - & {$[95]$} \\
\hline 97 & 369 & 493 & - & & 化学传感 & [95] \\
\hline
\end{tabular}

${ }^{a}$ Solution; ${ }^{b}$ Solid; -: Unreported.

少, 特别是磷光化合物的功能和应用研究偏少的情况, 进一步的研究, 可以充分利用含氟配体的多样性, 结合 中心金属在发光有机金属配合物中的作用和 AIPE 的特 点，进一步扩展有机金属配合物的种类，特别是合成更 多含有廉价金属如锌、铜等的配合物和磷光配合物. 结 合氟原子对有机金属配合物分子间作用力的影响，有可 能获得更多优异的性能, 如 MCL 及热致发光变色等刺 激响应性. 同时, 对于这些化合物体系发光机理的研究, 特别是结合理论计算以及超快光谱等手段的详细研究, 是必要和非常有用的, 对设计新型的发光有机金属配合 物体系有重要的指导意义.

尽管 AIE 含氟有机金属配合物的研究已取得一定 进展, 对于实际应用来说仍需要进一步深入研究. 例如, 利用 AIE 含氟有机金属配合物发光的高效、多样性和可 调节性, 拓展其在光电材料和器件领域的应用; 利用刺 激响应性特点, 拓展其在传感领域的应用; 利用磷光寿 命长等优点, 拓展其在生物成像等方面的应用. 相信在 不久的将来, 这个独特的研究方向, 会受到更多的重视, 涌现更多的成果.

\section{References}

[1] Joshi, K.; Singh, R.; Mishra, N.; Kumar, V.; Vinayak, V. ChemBioChem 2018, 19, 1630.

[2] Ma, D.; Ng, H.; Wong, S.; Vellaisamy, K.; Wu, K.; Leung, C. Dalton Trans. 2018, 47, 15278.

[3] Simpson, P.; Falasca, M.; Massi, M. Chem. Commun. 2018, 54, 12429

[4] Chen, S.; Pang, C.; Chen, X.; Yan, Z.; Huang, S.; Li, X.; Zhong, Y.; Wang, Z. Chin. J. Org. Chem. 2019, 39, 1846 (in Chinese). (陈思鸿, 庞楚明, 陈孝云, 严智浩, 黄诗敏, 李香弟, 钟雅婷, 汪朝阳，有机化学, 2019, 39, 1846.)

[5] Ren, B.; Yi, J.; Zhong, D.; Zhao, Y.; Guo, R.; Sheng, Y.; Sun, Y.; Xie, L.; Huang, W. Acta Chim. Sinica 2020, 78, 56 (in Chinese). (任保轶, 依建成, 钟道昆, 赵玉志, 郭闰达, 盛永刚, 孙亚光, 解令海, 黄维, 化学学报, 2020, 78, 56.)

[6] Chen, S.; Dao, J.; Zhou, K.; Luo, Y.; Su, S.; Pu, X.; Huang, Y.; Lu, Z. Acta Chim. Sinica 2017, 75, 367 (in Chinese).

(陈仕琦, 代军, 周凯峰, 罗艳菊, 苏仕健, 蒲雪梅, 黄艳, 卢志 云, 化学学报, 2017, 75, 367.)

[7] Luo, J.; Xie, Z.; Lam, J. W. Y.; Cheng, L.; Tang, B. Z.; Chen, H.; Qiu, C.; Kwok, H. S.; Zhan, X.; Liu, Y.; Zhu, D. Chem. Commun. 2001, 1740 .

[8] Chi, Z.; Zhang, X.; Xu, B.; Zhou, X.; Ma, C.; Zhang, Y.; Liu, S.; $\mathrm{Xu}, \mathrm{J}$. Chem. Soc. Rev. 2012, 41, 3878

[9] Chen, Z.; Liang, J.; Han, X.; Yin, J.; Yu, G.; Liu, S. Dyes Pigm. 2015, 112, 59 .
[10] Hariharan, P.; Venkataramanan, N.; Moon, D.; Anthony, S. J. Phys. Chem. C 2015, 119, 9460.

[11] Chen, M.; Sun, J.; Qin, A.; Tang, B. Z. Chin. Sci. Bull. 2016, 61, 304 (in Chinese) (陈明，孙景志，秦安军，唐本忠，科学通报, 2016, 61, 304.)

[12] Zhang, L.; Li, M.; Gao, Q.; Chen, C. Chin. J. Org. Chem. 2020, 40, 516 (in Chinese) (张亮, 李猛, 高庆宇, 陈传峰, 有机化学, 2020, 40, 516.)

[13] Mei, J.; Hong, Y.; Lam, J. W.; Qin, A.; Tang, Y.; Tang, B. Z. Adv. Mater. 2014, 26, 5429.

[14] Mei, J.; Leung, N. L.; Kwok, R. T.; Lam, J. W.; Tang, B. Z. Chem. Rev. 2015, 115, 11718

[15] Hong, Y.; Lam, J. W.; Tang, B. Z. Chem. Commun. 2009, 4332.

[16] Luo, J.; Song, K.; Gu, F. L.; Miao, Q. Chem. Sci. 2011, 2, 2029.

[17] Leung, N. L.; Xie, N.; Yuan, W.; Liu, Y.; Wu, Q.; Peng, Q.; Miao, Q.; Lam, J. W.; Tang, B. Z. Chem.-Eur. J. 2014, 20, 15349.

[18] Yao, L.; Zhang, S.; Wang, R.; Li, W.; Shen, F.; Yang, B.; Ma, Y. Angew. Chem., Int. Ed. 2014, 53, 2119.

[19] Liu, J.; Meng, Q.; Zhang, X.; Lu, X.; He, P.; Jiang, L.; Dong, H.; Hu, W. Chem. Commun. 2013, 49, 1199.

[20] Ravotto, L.; Ceroni, P. Coord. Chem. Rev. 2017, 346, 62.

[21] Alam, P.; Climent, C.; Alemany, P.; Laskar, I. J. Photochem. Photobiol., C 2019, 41, 100317.

[22] Jiang, B.; Zhang, C.; Shi, X.; Yang, H. Chin. J. Polym. Sci. 2019, 37,372 .

[23] Sathish, V.; Ramdass, A.; Thanasekaran, P.; Lu, K.; Rajagopal, S. J. Photochem. Photobiol., C 2015, 23, 25.

[24] Zhao, Q.; Li, L.; Li, F.; Yu, M.; Liu, Z.; Yi, T.; Huang, C. Chem. Commun. 2008, 685.

[25] Yang, X.; Yue, L.; Yu, Y.; Liu, B.; Dang, J.; Sun, Y.; Zhou, G.; Wu, Z.; Wong, W. Adv. Optical Mater. 2020, 8, 2000079.

[26] Chopra, D.; Row, T. CrystEngComm 2011, 13, 2175.

[27] Zhang, Q.; Kelly, M. A.; Bauer, N.; You, W. Acc. Chem. Res. 2017, $50,2401$.

[28] Babudri, F.; Farinola, G. M.; Naso, F.; Ragni, R. Chem. Commun. 2007, 1003.

[29] MilianMedina, B.; Gierschner, J. J. Phys. Chem. Lett. 2017, 8, 91.

[30] Liu, M.; Wu, Q.; Shi, H.; An, Z.; Huang, W. Acta Chim. Sinica 2018, 76, 246 (in Chinese).

(刘明丽, 吴琪, 史慧芳, 安众福, 黄维, 化学学报, 2018, 76, 246.)

[31] Xu, P.; Qiu, Q.; Ye, X.; Wei, M.; Xi, W.; Feng, H.; Qian, Z. Chem. Commun. 2019, 55, 14938.

[32] Qin, C.; Liu, W.; Nie, Y.; Gao, Y.; Miao, J.; Li, T.; Jiang, X. Chin. J. Org. Chem. 2020, 40, 2232 (in Chinese).

(秦成远, 刘威, 聂永, 高迎, 苗金玲, 李天瑞, 蒋绪川, 有机化 学, 2020, 40, 2232.)

[33] Lei, Z.; Pei, X.; Guan, Z.; Wang, Q. Angew. Chem., Int. Ed. 2017, 56,7117

[34] Wang, N.; Zhang, J.; Xu, X.; Feng, S. Dalton Trans. 2020, 49, 1883.

[35] Mo, L.; Jia, J.; Sun, L.; Wang, Q. Chem. Commun. 2012, 48, 8691.

[36] Guo, L. X.; Xing, Y.; Wang, M.; Sun, Y.; Zhang, X.; Lin, B. P.; Yang, H. J. Mater. Chem. C 2019, 7, 4828.

[37] Yan, X.; Wang, M.; Cook, T.; Zhang, M.; Saha, M.; Zhou, Z.; Li, X.; Huang, F.; Stang, P. J. Am. Chem. Soc. 2016, 138, 4580. 
[38] Krummacher, B.; Choong, V.; Mathai, M.; Choulis, S.; So, F.; Jermann, F.; Fiedler, T.; Zachau, M. Appl. Phys. Lett. 2006, 88, 113506.

[39] He, L.; Tan, C.; Cao, Q.; Mao, Z. Prog. Chem. 2018, 30, 1548 (in Chinese). (何良，谭彩萍，曹乾，毛宗万，化学进展, 2018, 30, 1548.)

[40] Zhang, X.; Xu, Y.; Shi, H. Prog. Chem. 2006, 18, 870 (in Chinese). (张秀菊, 许运华，史华红，化学进展, 2006, 18, 870.)

[41] Liao, Z.; Zhu, T.; Mi, B.; Gao, Z.; Fan, Q.; Huang, W. Prog. Chem. 2011, 23, 1627 (in Chinese) (廖章金, 朱䑣珺, 密保秀, 高志强, 范曲立, 黄维, 化学进展, 2011, 23, 1627.)

[42] You, Y.; Huh, H.; Kim, K.; Lee, S.; Kim, D.; Park, S. Chem. Commun. 2008, 3998.

[43] Shin, C. H.; Huh, J. O.; Baek, S. J.; Kim, S. K.; Lee, M. H.; Do, Y. Eur. J. Inorg. Chem. 2010, 3642.

[44] Alam, P.; Das, P.; Climent, C.; Karanam, M.; Casanova, D.; Choudhury, A. R.; Alemany, P.; Jana, N. R.; Laskar, I. R. J. Mater. Chem. C 2014, 2, 5615.

[45] Alam, P.; Kaur, G.; Climent, C.; Pasha, S.; Casanova, D.; Alemany, P.; Choudhury, A. R.; Laskar, I. R. Dalton Trans. 2014, 43, 16431.

[46] Song, Z.; Liu, R.; Li, Y.; Shi, H.; Hu, J.; Cai, X.; Zhu, H. J. Mater. Chem. C 2016, 4, 2553.

[47] Liu, R.; Song, Z.; Li, Y.; Li, Y.; Yao, W.; Yao, W.; Sun, H.; Zhu, H. Sens. Actuators, B 2018, 259, 840.

[48] Li, Y.; Ma, B.; Long, Y.; Song, Z.; Su, J.; Wang, Y.; Liu, R.; Song, G.; Zhu, H. J. Mater. Chem. C 2020, 8, 2467.

[49] Bejoymohandas, K. S.; George, T. M.; Bhattacharya, S.; Natarajan, S.; Reddy, M. L. P. J. Mater. Chem. C 2014, 2, 515.

[50] Liu, J.; Jiu, C.; Yuan, B.; Liu, X.; Chen, Y.; Ji, L.; Chao, H. Chem. Commun. 2017, 53, 2052.

[51] Qiu, K.; Ouyang, M.; Liu, Y.; Huang, H.; Liu, C.; Chen, Y.; Jia, L.; Chao, H. J. Mater. Chem. B 2017, 5, 5488.

[52] Darmawan, N.; Sambri, L.; Daniliuc, C. G.; De Cola, L. Dalton Trans. 2019, 48, 3664

[53] Xie, J.; Li, D.; Duan, Y.; Geng, Y.; Yang, T.; Li, G.; Zhu, D.; Su, Z. Dyes Pigm. 2020, 172, 107855.

[54] Shan, G.; Li, H.; Qin, J.; Zhu, D.; Liao, Y.; Su, Z. Dalton Trans. 2012, 41,9590 .

[55] Shan, G.; Li, H.; Sun, H.; Zhu, D.; Cao, H.; Su, Z. J. Mater. Chem. $C 2013,1,1440$

[56] Wu, Y.; Sun, H.; Cao, H.; Li, H.; Shan, G.; Duan, Y.; Geng, Y.; S, Z.; Liao, Y. Chem. Commun. 2014, 50, 10986.

[57] Li, G.; Ren, X.; Shan, G.; Che, W.; Zhu, D.; Yan, L.; Su, Z.; Bryce, M. R. Chem. Commun. 2015, 51, 13036.

[58] Zhao, K.; Mao, H.; Wen, L.; Shan, G.; Fu, Q.; Sun, H.; Su, Z. J. Mater. Chem. C 2018, 6, 11686.

[59] Wang, Y.; Yang, T.; Liu, X.; Li, G.; Che, W.; Zhu, D.; Su, Z. J. Mater. Chem. C 2018, 6, 12217.

[60] Che, W.; Li, G.; Liu, X.; Shao, K.; Zhu, D.; Su, Z.; Bryce, M. Chem. Commun. 2018, 54, 1730

[61] Congrave, D. G.; Batsanov, A. S.; Bryce, M. R. Dalton Trans. 2018, $47,16524$.

[62] Song, Z.; Liu, R.; Li, X.; Zhu, H.; Lu, Y.; Zhu, H. J. Mater. Chem. C 2018, 6, 10910 .

[63] Kim, H.; Jiang, H.; Choi, W.; Park, T.; Lee, J.; Bejoymohandas, K. J. Mater. Chem. C 2020, 8,4789 .

[64] Berenguer, J. R.; Lalinde, E.; Moreno, M. T. Coord. Chem. Rev. 2018, 366, 69 .

[65] Liu, S.; Sun, H.; Ma, Y.; Ye, S.; Liu, X.; Zhou, X.; Mou, X.; Wang, L.; Zhao, Q.; Huang, W. J. Mater. Chem. 2012, 22, 22167.
[66] Martinez-Junquera, M.; Lara, R.; Lalinde, E.; Moreno, M. J. Mater Chem. C 2020, 8, 7221.

[67] Ionescu, A.; Godbert, N.; Aiello, I.; Ricciardi, L.; La Deda, M.; Crispini, A.; Sicilia, E.; Ghedini, M. Dalton Trans. 2018, 47, 11645.

[68] Berenguer, J. R.; Lalinde, E.; Moreno, M. T.; Sanchez, S.; Torroba, J. Inorg. Chem. 2012, 51, 11665 .

[69] Shiotsuka, M.; Ono, R.; Kurono, Y.; Asano, T.; Sakae, Y. J. Organomet. Chem. 2019, 880, 116.

[70] Lu, W.; Chen, Y.; Roy, V.; Chui, S.; Che, C. Angew. Chem., Int. Ed. 2009, 48, 7621.

[71] Carrara, S.; Aliprandi, A.; Hogan, C.; De Cola, L. J. Am. Chem. Soc. 2017, 139, 14605.

[72] Wang, S.; Li, W.; Yu, Y.; Liu, J.; Zhang, C. Acta Phys.-Chim. Sin 2019, 35, 1276 (in Chinese).

(王士昭, 李维军, 俞越, 刘进, 张诚, 物理化学学报, 2019, 35, 1276.)

[73] Pintp, A.; Svahn, N.; Lima, J.; Robriguez, L. Dalton Trans. 2017, $46,11125$.

[74] Liang, J.; Chen, Z.; Yin, J.; Yu, G.; Liu, S. Chem. Commun. 2013, 49, 3567.

[75] Chen, Z.; Yang, L.; Hu, Y.; Wu, D.; Yin, J.; Yu, G.; Liu, S. RSC Adv. 2015, 5, 93757.

[76] Chen, Z.; Nie, Y.; Liu, S. RSC Adv. 2016, 6, 73933.

[77] Chen, Z.; Li, Z.; Hu, F.; Yu, G.; Yin, J.; Liu, S. Dyes Pigm. 2016 125,169

[78] Chen, Z.; Liu, G.; Pu, S.; Liu, S. Dyes Pigm. 2017, 143, 409.

[79] Li, W.; Luo, W.; Li, K.; Yuan, W.; Zhang, Y. Chin. Chem. Lett. 2017, 28, 1300 .

[80] Chen, Z.; Liu, G.; Pu, S.; Liu, S. Dyes Pigm. 2018, 159, 499

[81] Wang, X.; Zhang, J.; Dong, Y.; Zhang, Y.; Yin, J.; Liu, S. Dyes Pigm. 2018, 156, 74

[82] Liang, J.; Chen, Z.; Xu, L.; Wang, J.; Yin, J.; Yu, G.; Chen, Z. N.; Liu, S. J. Mater. Chem. C 2014, 2, 2243.

[83] Chen, Z.; Wu, D.; Han, X.; Liang, J.; Yin, J.; Yu, G.; Liu, S. Chem. Commun. 2014, 50, 11033.

[84] Chen, Z.; Han, X.; Zhang. J.; Wu, D.; Yu, G.; Yin, J.; Liu, S. RSC Adv. 2015, 5, 15341

[85] Chen, Z.; Zhang, J.; Song, M.; Yin, J.; Yu, G.; Liu, S. Chem. Commun. 2015, 51, 326.

[86] Song, M.; Chen, Z.; Yu, G.; Yin, J.; Liu, S. Chin. J. Org. Chem 2015, 35, 681 (in Chinese). (宋敏, 陈钊, 余广鳌, 尹军, 刘盛华, 有机化学, 2015, 35, 681.).

[87] Chen, Z.; Li, Z.; Yang, L.; Liang, J.; Yin, J.; Yu, G.; Liu, S. Dyes Pigm. 2015, 121, 170.

[88] Han, X.; Lu, X.; Chen, Z.; Yu, G..; Yin, J.; Liu, S. Chin. J. Chem. 2015, 33, 1064.

[89] Dong, Y.; Zhang, J.; Li, A.; Gong, J.; He, B.; Xu, S.; Yin, J.; Liu, S.; Tang, B. Z. J. Mater. Chem. C 2020, 8, 894.

[90] Zhang, J.; Liu, Q.; Wu, W.; Peng, J.; Zhang, H.; Song, F.; He, B. Wang, X.; Wang, X.; Sung, H.; Liu, S.; Li, B.; Lam, J.; Tang, B. Z. ACS Nano 2019, 13, 3618.

[91] Zhang, J.; He, B.; Wu, W.; Alam, P.; Zhang, H.; Gong, J.; Song, F.; Wang, Z.; Sung, H.; Williams, I.; Wang, Z.; Lam, J.; Tang, B. Z. J. Am. Chem. Soc. 2020, 142, 14608.

[92] Chen, Z.; Liu, G.; Pu, S.; Liu, S. Dyes Pigm. 2018, 152, 54

[93] Chen, Z.; Huang, P.; Li, Z.; Yin, J.; Yu, G.; Liu, S. Inorg. Chim. Acta 2015, 432, 192

[94] Li, J.; Wu, P.; Jiang, W.; Li, B.; Wang, B.; Zhu, H.; Roesky, H. Angew. Chem., Int. Ed. 2020, 59, 10027.

[95] Bandrowsky, T. L.; Carroll, J. B.; Braddock-Wilking, J. Organometallics 2011, 30, 3559 . 